\begin{tabular}{|c|c|}
\hline Title & How waves and turbulence maintain the super-rotation of $V$ enus' atmosphere \\
\hline Author(s) & $\begin{array}{l}\text { Horinouchi, Takeshi; Hay ashi, Y oshi-Y uki; W atanabe, Shigeto; Y amada, Manabu; Y amazaki, A tsushi; Kouyama, } \\
\text { Toru; T aguchi, Makoto; Fukuhara, Tetsuya; Takagi, Masahiro; Ogohara, Kazunori; Murakami, Shin-ya; Peralta, Javier; } \\
\text { Limaye, Sanjay S.; Imamura, T akeshi; Nakamura, Masato; Sato, T akao M.; Satoh, Takehiko }\end{array}$ \\
\hline Citation & $\begin{array}{l}\text { Science, 368(6489), 405-409 } \\
\text { https://doi.org/10.1126/science.aaz4439 }\end{array}$ \\
\hline Issue Date & $2020-0424$ \\
\hline Doc URL & http://hdl.handle.net/2115/78661 \\
\hline Rights & $\begin{array}{l}\text { This is the author' sversion of the work. It is posted here by permission of the A A A S for personal use, not for } \\
\text { redistribution. The definitive version was published in Science, vol.368 on } 24 \text { A pr 2020, DOI: } 10.1126 / \text { science.aaz } 4439\end{array}$ \\
\hline Type & article (author version) \\
\hline File Information & A katsuki_SRdiag_Science_ms5th.pdf \\
\hline
\end{tabular}

Instructions for use 


\section{How waves and turbulence maintain the super-rotation of Venus' atmosphere}

Takeshi Horinouchi $^{1,2 *}$, Yoshi-Yuki Hayashi ${ }^{3}$, Shigeto Watanabe ${ }^{4}$, Manabu Yamada ${ }^{5}$, Atsushi Yamazaki $^{2}$, Toru Kouyama ${ }^{6}$, Makoto Taguchi ${ }^{7}$, Tetsuya Fukuhara ${ }^{7}$, Masahiro Takagi ${ }^{8}$, Kazunori Ogohara $^{9}$, Shin-ya Murakami ${ }^{2}$, Javier Peralta ${ }^{2}$, Sanjay S. Limaye ${ }^{10}$, Takeshi Imamura ${ }^{11}$, Masato Nakamura $^{2,12}$, Takao M. Sato ${ }^{4}$, Takehiko Satoh ${ }^{2,12}$

${ }^{1}$ Faculty of Environmental Earth Science, Hokkaido University, Sapporo, Japan

2 Institute of Space and Astronautical Science, Japan Aerospace Exploration Agency, Sagamihara, Japan

${ }^{3}$ Department of Planetology / Center for Planetary Science, Kobe University, Kobe, Japan

${ }^{4}$ Space Information Center, Hokkaido Information University, Ebetsu, Japan

${ }^{5}$ Planetary Exploration Research Center, Chiba Institute of Technology, Narashino, Japan

${ }^{6}$ Artificial Intelligence Research Center, National Institute of Advanced Industrial Science and Technology, Tokyo, Japan

$15 \quad{ }^{7}$ College of Science, Rikkyo University, Tokyo, Japan

${ }^{8}$ Faculty of Science, Kyoto Sangyo University, Kyoto, Japan

${ }^{9}$ School of Engineering, University of Shiga Prefecture, Hikone, Japan

${ }^{10}$ Space Science and Engineering Center, the University of Wisconsin-Madison, Madison, WI, USA

${ }^{11}$ Graduate School of Frontier Sciences, the University of Tokyo, Kashiwa, Japan

12 Department of Space and Astronautical Science, School of Physical Sciences, The Graduate University for Advanced Studies, SOKENDAI, Tokyo, Japan

* email: horinout@ees.hokudai.ac.jp

Abstract: Venus has a thick atmosphere which rotates 60 times as fast as the surface, a phenomenon known as super-rotation. We use data from the orbiting Akatsuki spacecraft to investigate how the super-rotation is maintained in the cloud layer, where the rotation speed is highest. A thermally induced latitudinal-vertical circulation acts to homogenize the distribution of the angular momentum around the rotational axis. Maintaining the super-rotation requires this to be counteracted by atmospheric waves and turbulence. Among those effects, thermal tides transport the angular momentum that maintains the rotation peak near the cloud top at low latitude. Other planetary-scale waves and large-scale turbulence act in the opposite direction. We suggest that hydrodynamic instabilities adjust the angular-momentum distribution at midlatitudes.

\section{Main Text:}

Venus' thick atmosphere rotates at speeds up to 60 times the slow planetary rotation (the surface rotates westwards with a period of 243 days; throughout this paper, "day" refers to Earth days). The mechanism responsible for this super-rotation (SR) remains unclear $(1,2)$, partly due to insufficient observational evidence for hypothesis testing. 
SR may also occur in tidally-locked exoplanets i.e. those which always face the same way towards their host star, so are heated only on one side. Zonal (east-west) flow around the rotation axis, including SR, can transport heat from the dayside to the nightside of those exoplanets (3).

SR cannot occur unless the atmospheric angular momentum with respect to the rotational axis (hereinafter, AM) is transported by axially asymmetric flows ("eddies") such as waves and turbulence. Without them, AM (per unit mass) is homogenized by the latitudinal-vertical circulation (meridional circulation) induced by the pole-equator temperature difference (4). Attempts to estimate the AM transport from observations were made by tracking clouds using images of Venus' cloud top, which is at $65-70 \mathrm{~km}$ altitude, obtained by the Mariner 10 (5) and Pioneer Venus Orbiter $(6,7)$ spacecraft, but the sampling proved insufficient both in space and time.

Simulations with atmospheric general circulation models have generated SR (8-15). These studies indicate that SR is realized by AM transport by tidal $(9,11,13,14)$ and other waves $(8,10$ 15), some of which arising from hydrodynamic instabilities [such as those called barotropic $(10,12-14)$, baroclinic $(12,13,15)$, and Rossby-Kelvin instabilities (14)], and turbulent eddies (15). The relative contributions of each of these effects to SR differ between the models.

The AM budget can be described with the following equation, which is called the transformed Eulerian mean equation in meteorology,

$$
\frac{\partial M}{\partial t}=-\boldsymbol{v}^{r} \cdot \boldsymbol{\nabla} M+\rho_{0}^{-\mathbf{1}} \boldsymbol{\nabla} \cdot \boldsymbol{F},
$$

where $M$ is the zonal-mean (i.e., averaged along latitudinal circles) AM per unit mass, which is negative when westward as in SR, $\boldsymbol{F}$ is (minus) the AM flux, called the Eliassen-Palm flux, $\boldsymbol{v}^{r} \equiv$ $\left(0, v^{r}, w^{r}\right)$ represents the mean meridional circulation velocities (16). Equation (1) indicates that, at steady state, AM transport by "eddies" (the $\rho_{0}^{-\mathbf{1}} \boldsymbol{\nabla} \cdot \boldsymbol{F}$ term) and that by the mean meridional circulation (the $-\boldsymbol{v}^{r} \cdot \boldsymbol{\nabla} M$ term) compensate each other. On Venus, $|M|$ reaches its maximum around the cloud top altitude near the equator $(17,18)$. Maintenance of the maximum requires eddy AM transport to that region.

We begin by briefly examining the mean meridional circulation and its effect. To determine the circulation directly from wind observations is difficult even for the Earth's atmosphere. For Venus, the most credible estimates are provided by observations of solar and thermal radiations, from which we constructed a model (10). The obtained $w^{r}$ and $v^{r}$ indicate upwelling of a few $\mathrm{mm} \mathrm{s}^{-1}$ in the equatorial upper cloud layer and poleward flow on the order of $1 \mathrm{~m} \mathrm{~s}^{-1}$ at around the mid-latitude cloud top, respectively (Fig. 1).

We introduce an idealized zonal wind (eastward velocity component) structure into the model (16). The upwelling in the equatorial cloud layer acts to decelerate the SR by of $\sim 1 \mathrm{~m} \mathrm{~s}^{-1} \mathrm{day}^{-1}$ at the AM peak around the equatorial cloud top (Fig 1C), which needs to be compensated by eddy acceleration.

At mid-to-high latitudes, negative (positive) acceleration occurs through the $-\boldsymbol{v}^{r} \cdot \boldsymbol{\nabla} M$ term above (below) $\sim 65 \mathrm{~km}$ (Fig. 1C). We expect that hydrodynamic instabilities induced inevitably 
by the circulation $(19,20)$ compensate for these accelerations $(16$, Fig. S2), so we infer that the AM distribution at mid-to-high latitudes can be formed more-or-less spontaneously.

We provide further constraints by data from the Akatsuki spacecraft orbiting Venus. We analyze observations taken between December 2015 and December 2018, divided into five subperiods, starting from 2015-12-07, 2016-09-21, 2017-04-21, 2017-12-01, and 2018-06-16 (ending on 2018-12-07). Using a cloud-tracking method with quality control $(21,22)$, we derived wind estimates using Akatsuki's Ultraviolet Imager (UVI) (23) at the wavelengths of 365 and $283 \mathrm{~nm}$. We derived horizontal velocities near the cloud top over regions of $\sim 1,000 \mathrm{~km} \times 1,000 \mathrm{~km}$ with a time resolution of four hours (16) with uncertainties shown in Fig. S3A. The 283-nm results capture winds at slightly higher altitude than the 365 -nm results, presumably by a few km (24).

The meridional component of the EP flux $F_{y}$ is likely dominated by the $f_{y v} \equiv-\left\langle u^{*} v^{*}\right\rangle \cos \varphi$ term (16), where $u$ and $v$ are zonal and meridional (south-to-north) winds, respectively, and angle brackets and asterisk represent zonal mean and the deviation from it, respectively. We employ a coordinate system based on local time $\tau_{L}$, latitude $\varphi$, and time $t$, where $\tau_{L}$ is defined by assigning $0-24 \mathrm{~h}$ to longitudes relative to the anti-solar point. Temporally averaged winds on this system $(\bar{u}, \bar{v})$ consist of zonal and temporal mean winds $\left(u_{0}, v_{0}\right)$ and the flow associated with the thermal tides $\left(u_{t}, v_{t}\right)$. The mean winds from the three years are shown in Fig. S3.

We estimated $\left(u_{t}, v_{t}\right)$ by subtracting the model estimated $\left(u_{0}, v_{0}\right)$ from the observationally derived $(\bar{u}, \bar{v})$ for each of UVI image subperiods (16). The resulting tidal AM flux, $\left\langle u_{t} v_{t}\right\rangle \cos \varphi$, is shown in Fig. 2A. It is positive (negative) in the northern (southern) hemisphere, indicating equatorward transport of westward AM in the both hemispheres. The consistency between our results for different subperiods (Fig 2A) demonstrates the robustness of our estimation. The tidal meridional AM flux convergence acts to accelerate the SR between $20^{\circ} \mathrm{S}$ and $20^{\circ} \mathrm{N}$, reaching peak values around $-0.6(-0.9) \mathrm{m} \mathrm{s}^{-1}$ day $^{-1}$ for the $365-\mathrm{nm}(283-\mathrm{nm})$ case, indicating the acceleration of westward SR (Fig. 2C). The estimates depend on $u_{0}$ and $v_{0}$; we performed a sensitivity study (16, Figs. S9-10) to verify the robustness of the estimates, although some uncertainty remains as described in the supplementary document (16). Thermal tide carries eastward AM, so the present result is consistent with the action-reaction law on the tide generation by solar heating stronger at lower latitude.

We next examine the AM transport by transient motions other than thermal tides. We use $\left(u^{\prime}\right.$, $\left.v^{\prime}\right) \equiv(u-\tilde{u}, v-\tilde{v})$, where $(\tilde{u}, \tilde{v})$ are the averages of $(u, v)$ over \pm 15 days at fixed $\tau_{\mathrm{L}}$ and $\varphi$. Figure $2 \mathrm{~B}$ shows the AM flux $\overline{\left\langle u^{\prime} v^{\prime}\right\rangle} \cos \varphi$ (solid lines) after the effects of the correlation between the errors in $u^{\prime}$ and $v^{\prime}$ have been subtracted by using the error covariance (16, Fig. S5). Prior to subtraction, the error covariance enhanced the AM flux by a factor of 1.5 times. However, this effect is very small (10) for $\overline{\left\langle u^{\#} v^{\#}\right\rangle} \cos \varphi$, where $\left(u^{\#}, v^{\#}\right)$ is $\left(u^{\prime}, v^{\prime}\right)$ smoothed by "daily averaging", which is time averaging over each calendar day at fixed $\tau_{L}$ and $\varphi$. UVI was typically operated for 16 hours each day, so the daily averaging smooths winds over several thousand $\mathrm{km}$ in longitude because of the SR.

The derived AM flux (Fig. 2B) is opposite to that provided by the thermal tides (Fig. 2A). The consistency between subperiods again indicates the robustness of our method. Unlike the tidal case, both wavelengths produce quantitatively consistent results, suggesting a deceleration of the 
SR weaker than the tidal acceleration (Fig. 2C). The AM flux computed with $\left(u^{\#}, v^{\#}\right)$ (Fig. 2B) is slightly weaker than that computed with $\left(u^{\prime}, v^{\prime}\right)$, suggesting that a small fraction of the AM flux resides at high frequencies (periods shorter than 2 days) or, equivalently, at small scales.

Next we consider the nature of transient disturbances and their roles in the AM transport. Figure $3 \mathrm{~A}$ presents an example of the spatial distribution of wind disturbances using $\left(u^{\#}, v^{\#}\right)$ and the absolute vorticity computed with $\left(u^{\#}+\tilde{u}, v^{\#}+\tilde{v}\right)$, constructed by shifting by $90^{\circ}$ per day, approximating the advection by the SR; more examples are shown in Figs. S6-S7. The smooth transition over adjacent days indicates that overall temporal evolution is slow. The distribution of $u^{\#}$ suggests the dominance of variability at zonal wavenumber (the wavenumber along longitude in radian) equal to 1 (i.e, single wave extending over latitudinal circles), consistent with the waves called Rossby waves $(25,26)$. We derived power spectra of $u^{\#}$ (Fig. 3B) which reach a maximum at frequency around 0.2 day $^{-1}$, corresponding to a ground-based period of 5.2 days, where the sign of the imaginary part of cross-spectrum (Fig. S8C) arising from quadrature-phase lags is consistent with the Rossby wave structure. A secondary spectral peak appears in Fig. 3B at 0.24 day $^{-1}$ (corresponding to a ground-based period of 4 days), which is consistent with the waves called Kelvin waves $(25,26)$. The equivalent signal in $v^{\#}$ away from the equator (Fig. S8B) indicates a distortion absent in pure Kelvin waves.

In addition to the waves, turbulence-like motion is apparent in Fig. 3A (and further in Fig. S6). The non-monotonic change of absolute vorticity with latitude around $30^{\circ} \mathrm{N}$ indicates overturning motion to generate turbulence. Meandering (often cross-equatorial) flows prevail near the equator where the directions of absolute-vorticity gradients vary substantially, which is difficult to explain with simple superpositions of Rossby waves. These flows are also unlike gravity waves. Therefore, we conclude that some portion of the transient wind disturbances are due to horizontal turbulence.

The real part of the cross-spectrum (co-spectrum) of $u^{\#}$ and $v^{\#}$ decomposes their correlation along frequency (Fig. 3C). The signs at different frequencies almost agree at latitudes poleward of $20^{\circ} \mathrm{N}$ and $20^{\circ} \mathrm{S}$. The co-spectrum of two random signals has an equal probability to be positive or negative, so this agreement cannot be explained by coincidence or by noise. From the cospectra, we computed the contribution of the Rossby-wave periods (frequencies $0.1888-0.2222$ day $^{-1}$ ) to the meridional AM flux (Fig. 2C). This is only a small fraction of the AM transport, at least between $30^{\circ} \mathrm{S}$ and $30^{\circ} \mathrm{N}$. The contribution of the Kelvin waves is even smaller. We suggest that horizontal turbulence at broad frequency (together with waves) collectively decelerates the SR. This is opposite to the theoretical expectation that turbulent AM transport accelerates SR, which is required in the mechanism called the classical Gierasch-Rossow-Williams mechanism in which AM transport by meridional circulation and that by turbulence balance $(1,27,28)$.

We further investigate the vertical component of $\boldsymbol{F}, F_{z} \propto f_{z w}+f_{z T}$, where $f_{z w}$ and $f_{z T}$ are terms proportional to $\left\langle u^{*} w^{*}\right\rangle$ and $\left\langle v^{*} T^{*}\right\rangle$, respectively (16); here, $w$ is vertical velocity. We estimated the meridional heat flux by the diurnal (i.e., zonal-wavenumber 1) components of the thermal tides (diurnal tides), $\left\langle v_{t} T_{t}\right\rangle_{1} \propto f_{z T}$, by using the brightness temperature obtained from Akatsuki's Long Infrared camera LIR (16). This shows that the tidal heat flux is poleward (Fig. 2D). The diurnal tides are dominated by a Rossby wave at mid-to-high latitude (29). Theoretically, the $F_{z}$ 
component of a Rossby wave is dominated by $f_{z T}$, so its estimation (Fig.2E) indicates that the wave transports negative AM downward. Its typical magnitude is $1 \times 10^{-2} \mathrm{~m}^{2} \mathrm{~s}^{-2}$ at mid latitudes. This indicates that its effect on SR is minor, because the expected acceleration by that is $\sim 0.1 \mathrm{~m} \mathrm{~s}^{-1}$ day $^{-1}$ (this particular value follows if the vertical convergence of $f_{z T}$ due to tidal excitation occurs over a height scale of $10 \mathrm{~km}$ ).

The entire semidiurnal (i.e., zonal-wavenumber 2) components of the thermal tides (semidiurnal tides) and the low-latitude portion of the diurnal tides are dominated by gravity waves (29). They are expected to transport AM to its upgradient to accelerate the SR $(9,30)$. Observational estimation of $F_{z}$ associated with gravity waves are not available, because it is dominated by $f_{z w}$ so includes vertical velocity. We instead performed an order-of-magnitude estimation (16), finding that the acceleration associated with $\frac{\partial F_{z}}{\partial z}$ is likely $\sim 1 \mathrm{~m} \mathrm{~s}^{-1} \mathrm{day}^{-1}$ or smaller. Therefore, the tidal vertical AM transport can contribute to the acceleration of the SR at low latitude by a comparable amount to the horizontal transport.

The Akatsuki data have allowed us to estimate the horizontal AM transport by thermal tides, planetary-scale Rossby waves, and other transient disturbances. The thermal tides transport westward (super-rotating) AM equatorward, contributing to the maintenance of the SR against the homogenization by the meridional circulation. The other motions act in the opposite direction at low latitudes. We also estimated vertical AM transport by thermal tides. The AM balance governing the maintenance of the SR in the cloud layer is schematically illustrated in Fig. 4. We suggest that the SR maintenance mechanism is a version of the non-classical Gierasch-RossowWilliams scenario (1), which extends the classical one to include AM transport by tidal waves.

\section{References}

1. A. Sánchez-Lavega, S. Lebonnois, T. Imamura, P. Read, D. Luz, The atmospheric dynamics of Venus. Space Science Reviews 212(3-(4), 1541-1616 (2017).

2. P. L. Read, S. Lebonnois. Superrotation on Venus, on Titan, and elsewhere. Annual Review of Earth and Planetary Sciences, 46, 175-202 (2018).

3. A. P. Showman, L. M. Polvani, Equatorial superrotation on tidally locked exoplanets. The Astrophysical Journal 738(1), 71 (2011).

4. R. Hide, Dynamics of the atmospheres of the major planets with an appendix on the viscous boundary layer at the rigid bounding surface of an electrically-conducting rotating fluid in the presence of a magnetic field. J. Atmos. Sci., 26, 841-853 (1969).

5. S.S. Limaye, V.E. Suomi, Cloud motions on Venus - Global structure and organization. $J$. Atmos. Sci. 38, 1220-1235 (1981).

6. W. B. Rossow, A. D. del Genio, T. Eichler. Cloud-tracked winds from Pioneer Venus OCPP images, J. Atmos. Sci., 47(17), 2053-2084 (1990).

7. S. S. Limaye, Venus atmospheric circulation: Known and unknown, J. Geophys. Res., 112, E04S09, doi:10.1029/2006JE002814 (2007).

8. M. Yamamoto, M. Takahashi, The fully developed superrotation simulated by a general circulation model of a Venus-like atmosphere. J. Atmos. Sci., 60(3), 561-574 (2003).

9. M. Takagi, Y. Matsuda, Effects of thermal tides on the Venus atmospheric superrotation. $J$. Geophys. Res., D09112, doi:10.1029/2006JD007901 (2007).

10. C. Lee, S. R. Lewis, P. L. Read, Superrotation in a Venus general circulation model, J. Geophys. 
Res., 112, E04S11, doi:10.1029/2006JE002874 (2007).

11. S. Lebonnois, F. Hourdin, V. Eymet, A. Crespin, R. Fournier, F. Forget, Superrotation of Venus' atmosphere analyzed with a full general circulation model, J. Geophys. Res., 115, E06006, doi:10.1029/2009JE003458 (2010).

12. N. Sugimoto, M. Takagi, Y. Matsuda, Waves in a Venus general circulation model, Geophys. Res. Lett., 41, 7461-7467, doi:10.1002/2014GL061807 (2014).

13. S. Lebonnois, N. Sugimoto, G. Gilli, Wave analysis in the atmosphere of Venus below 100-km altitude, simulated by the LMD Venus GCM. Icarus, 278, 38-51 (2016).

14. J. M.Mendonça, P. L. Read, Exploring the Venus global super-rotation using a comprehensive general circulation model. Planetary and space science, 134, 1-18.(2016).

15. N. Sugimoto, M. Takagi,Y. Matsuda, Fully developed superrotation driven by the mean meridional circulation in a Venus GCM. Geophysical Research Letters, 46, 1776-1784 (2019).

\section{Supplementary materials of this paper to be available at the Science website}

17. G. Schubert, et al., Structure and circulation of the Venus atmosphere. J. Geophys. Res., 85(A13), 8007-8025 (1980).

18. V. V. Kerzhanovich, S. S. Limaye, Circulation of the atmosphere from the surface to $100 \mathrm{~km}$. Advances in Space Research, 5(11), 59-83 (1985).

19. S. I. Iga,Y.,Matsuda, Shear instability in a shallow water model with implications for the Venus atmosphere. J. Atmos. Sci., 62(7), 2514-2527 (2005).

20. M. Takagi, Y. Matsuda, A study on the stability of a baroclinic flow in cyclostrophic balance on the sphere. Geophysical research letters, 33, L14807, doi:10.1029/2006GL026200 (2006).

21. S. Ikegawa, T. Horinouchi, Improved automatic estimation of winds at the cloud top of Venus using superposition of cross-correlation surfaces. Icarus, 271, 98-119(2016).

22. T. Horinouchi, S. Murakami, T. Kouyama, K. Ogohara, A. Yamazaki, M. Yamada, S. Watanabe, Image velocimetry for clouds with relaxation labeling based on deformation consistency. Meas. Sci. Technol., 28(8), 085301. doi:10.1088/1361-6501/aa695c (2017).

23. A. Yamazaki, et al. Ultraviolet imager on Venus orbiter Akatsuki and its initial results. Earth, Planets and Space, 70(1), 23. doi:10.1186/s40623-017-0772-6 (2018).

24. T. Horinouchi et al., Mean winds at the cloud top of Venus obtained from two-wavelength UV imaging by Akatsuki. Earth, Planets and Space, 70:10, https://doi.org/10.1186/s40623-0170775-3 (2018).

25. T. Kouyama, T. Imamura, M. Nakamura, T. Satoh, Y. Futaana, Long-term variation in the cloud-tracked zonal velocities at the cloud top of Venus deduced from Venus Express VMC images. J. Geophys. Res. 118, 37-46 (2013).

26. M. Imai et al., Planetary-scale variations in winds and UV brightness at the Venusian cloud top: Periodicity and temporal evolution. J. Geophys. Res. Planets, 124, 2635-2659. https://doi.org/10.1029/2019JE006065 .

27. P. J. Gierasch, Meridional circulation and the maintenance of the Venus atmospheric rotation. J. Atmos. Sci., 32(6), 1038-1044 (1975).

28. W. B. Rossow, G. P. Williams, Large scale motions in the Venus stratosphere. J. Atmos. Sci. 36, 377-389 (1979).

29. T. Kouyama et al. Global structure of thermal tides in the upper cloud layer of Venus revealed by the LIR onboard Akatsuki. Geophys. Res. Lett., 46, 9457-9465. https://doi.org/10.1029/2019GL083820 (2019).

30. M. Newman, C. Leovy, Maintenance of strong rotational winds in Venus' middle atmosphere by thermal tides. Science, 257(5070), 647-650 (1992). 
31. S. Murakami, et al., Venus Climate Orbiter Akatsuki UVI Longitude-Latitude Map Data. doi:10.17597/isas.darts/vco-00016 (2018).

32. S. Murakami, et al., Venus Climate Orbiter Akatsuki LIR Longitude-Latitude Map Data. doi:10.17597/isas.darts/vco-00019 (2018).

33. S. Murakami, et al., Venus Climate Orbiter Akatsuki IR2 Longitude-Latitude Map Data. doi:10.17597/isas.darts/vco-00018 (2018).

34. M. Nakamura, et al., Overview of Venus orbiter, Akatsuki. Earth, planets and space, 63(5), 443-457 (2011).

35. M. Nakamura, et al., AKATSUKI returns to Venus. Earth Planets Space, 68:75, doi:10.1186/s40623-016-0457-6 (2016).

36. T. Horinouchi, et al., Equatorial jet in the lower to middle cloud layer of Venus revealed by Akatsuki. Nature Geoscience, 10, 646-651. doi:10.1038/ngeo3016 (2017)

37. J. Peralta, et al., Nightside Winds at the Lower Clouds of Venus with Akatsuki/IR2: Longitudinal, Local Time, and Decadal Variations from Comparison with Previous Measurements. The Astrophysical Journal Supplement Series, 239(2), 29 (2018).

38. M. G. Tomasko, L. R. Doose, P. H. Smith, A. P. Odell,. Measurements of the flux of sunlight in the atmosphere of Venus. J. Geophys. Res., 85(A13), 8167-8186 (1980).

39. V. I. Moroz, et al., Solar and thermal radiation in the Venus atmosphere. Advances in space research, 5, 197-232 (1985).

40. D. G.Andrews, J. R. Holton, C. B. Leovy, Middle Atmosphere Dynamics. (Academic press, Orland, Florida, 1987).

41. R. Hueso, J. Peralta, A. Sánchez-Lavega, Assessing the long-term variability of Venus winds at cloud level from VIRTIS-Venus Express. Icarus, 217(2), 585-598 (2012).

42. W. B. Rossow, A. D. Del Genio, S. S. Limaye, L. D. Travis, P. H. Stone, Cloud morphology and motions from Pioneer Venus images. J. Geophys. Res., 85(A13), 8107-8128 (1980).

43. F. W. Taylor, R. Beer, T. Chahine, D. J. Diner, L. S. Elson, R. D. Haskins, D. J. McCleese, J. V. Martonchik, P. E. Reichley, Structureand Meteorology of the Middle Atmosphere of Venus: Infrared Remote Sensing Fromthe Pioneer Orbiter. Journal of Geophysical Research, 85, 79638006 (1980).

44. M. Takagi, N. Sugimoto, H. Ando, Y. Matsuda, Three-dimensional structures of thermal tides simulated by a Venus GCM. J. Geophys. Res.: Planets, 123, 335-352 (2018).

45. M. Yamamoto, K. Ikeda, M. Takahashi, T. Horinouchi, Solar-locked and geographical atmospheric structures inferred from a Venus general circulation model with radiative transfer. Icarus, 321, 232-250 (2019).

46. J. D. Scargle, Studies in astronomical time series analysis. II - Statistical aspects of spectral analysis of unevenly spaced data. The Astrophysical Journal, 263, 835. https://doi.org/10.1086/160554 (1982).

40 47. T. Fukuhara, et al., LIR: Longwave infrared camera onboard the Venus orbiter Akatsuki. Earth, Planets and Space, 63(9), 1009-1018 (2011).

48. N. I.Ignatiev, et al. Altimetry of the Venus cloud tops from the Venus Express observations. $J$. Geophys. Res., 114, E00B43, doi:10.1029/2008JE003320 (2008).

49. D. Crisp, Radiative forcing of the Venus mesosphere: II. thermal fluxes, cooling rates, and radiative equilibrium temperatures. Icarus, 77(2), 391-413 (1989).

50. D. Crisp, D. Titov, "The thermal balance of the Venus atmosphere." in Venus II, S. W. Bougher, 
D. M. Hunten, R. J. Phillips, Eds. (University of Arizona Press, Tucson, Arizona, 1997), pp 353-384.

51. Y. J.Lee, D. V. Titov, N. I. Ignatiev, S. Tellmann, M. Pätzold, G. Piccioni, The radiative forcing variability caused by the changes of the upper cloud vertical structure in the Venus mesosphere. Planetary and Space Science, 113, 298-308 (2015).

52. S.S. Limaye, Venus: Cloud level circulation during 1982 as determined from Pioneer cloud photopolarimeter images. II - Solar longitude dependent circulation. Icarus 73, 212-226 (1988).

53. A. Seiff, et al., Models of the structure of the atmosphere of Venus from the surface to 100 kilometers altitude. Advances in Space Research, 5(11), 3-58 (1985).

54. D. O. Staley, The adiabatic lapse rate in the Venus atmosphere. J. Atmos. Sci., 27(2), 219-223 (1970).

55. A. Y. Hou, R. M. Goody, Diagnostic requirements for the superrotation on Venus. J. Atmos. Sci., 42(5), 413-432 (1985).

56. M. S. Longuet-Higgins, The eigenfunctions of Laplace's tidal equation over a sphere. Philosophical Transactions of the Royal Society of London. Series A, 262(1132), 511-607 (1968).

\section{Acknowledgements}

We thank our numerous colleagues who supported the Akatsuki project. We also thank the three anonymous reviewers for providing comments that helped improve this paper. Funding: This study was partially supported by the JSPS grant-in-aid 16H02231, 16H02225, 19H05605, 19K14789 and by NASA Grant NNX16AC79G. JP acknowledges JAXA's International Top Young Fellowship. Author contributions: TH derived the wind estimations, conducted analysis, produced the figures, and wrote the text. KO, SM, MT, TK, and TH developed the cloudtracking program. YYH, JP, SSL, and TS contributed to the interpretation. SW, MY, and AY calibrated the UVI radiance. TK, MT, and TF derived the temperature using LIR. KO and MT refined the geographic data mapping. TMS, TS, TI, SM and MN coordinated Akatsuki's observations and processed the original data. Competing interests: The authors have no competing interests. Data availability: The Akatsuki data are available online $(31,32,33)$. The data used in this paper, which include cloud tracking results and data visualized in figures are available online at https://darts.isas.jaxa.jp/pub/akatsuki/paper/Horinouchi_2020/ and in the supplementary materials (16). 


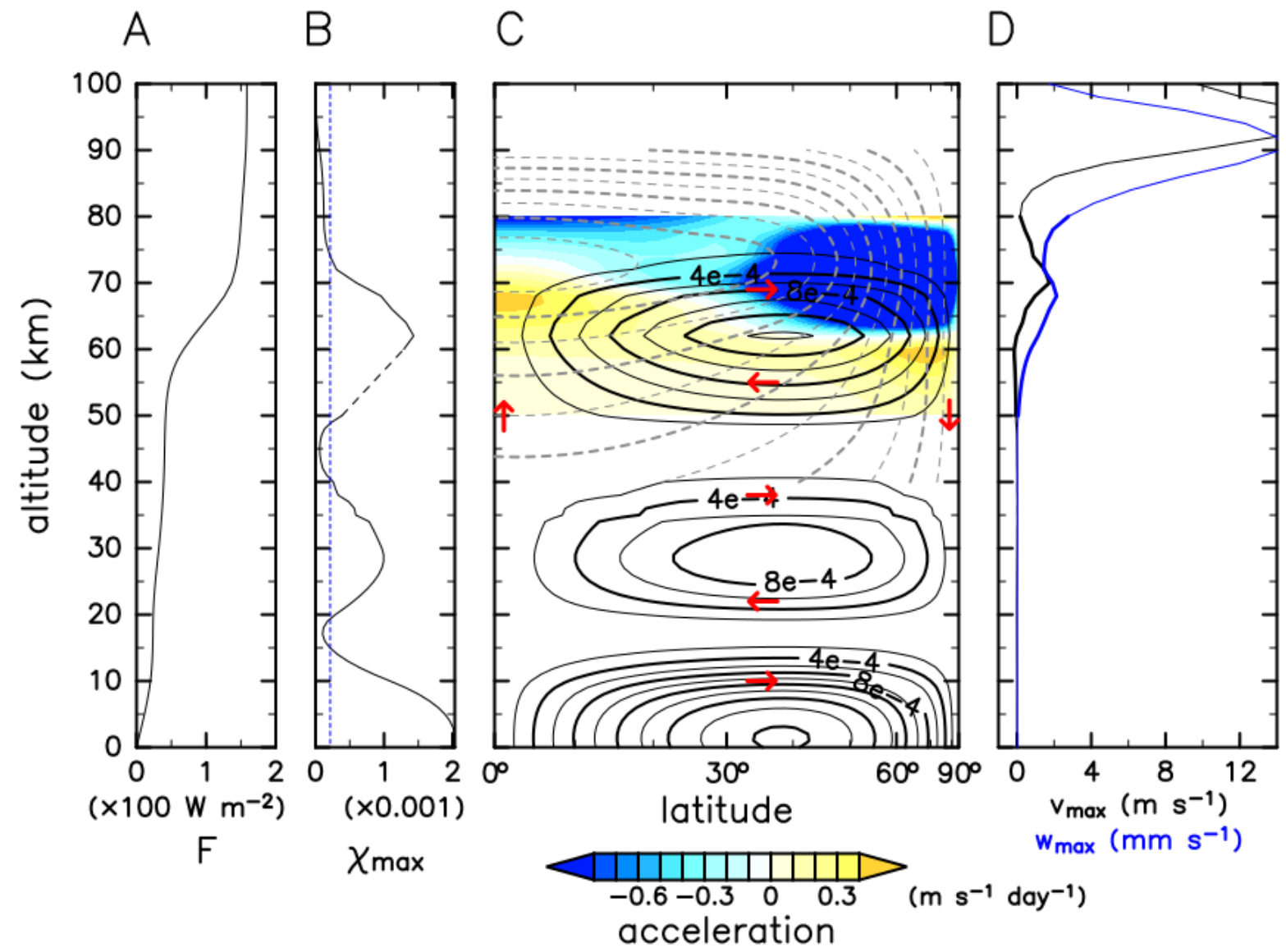

Fig. 1. Idealized model calculation of the meridional circulation derived from solar heating and the advective acceleration of idealized zonal AM (16). (A) Global-mean downward solar flux. (B) The mass stream function $\chi$ derived from the solar heating at $\varphi_{\max }=38.2^{\circ}$. Dashed line indicates interpolation. Blue dotted line indicates the value at $74 \mathrm{~km}$. (C) Hemispheric structure of $\chi$ (black contours; flow directions are indicated schematically by red arrows); idealized $M$ between 40 and $90 \mathrm{~km}$ (grey dashed contours); advective zonal acceleration $-(a \cos \varphi)^{-1} \boldsymbol{v}^{r} \cdot \boldsymbol{\nabla} M$ between 50 and $80 \mathrm{~km}$ (color shading). (D) $v^{r}$ at $\varphi_{\text {max }}$ (black curve) and $w^{r}$ at the equator (blue curve). Values between 50 and $80 \mathrm{~km}$ are highlighted by thickening. 


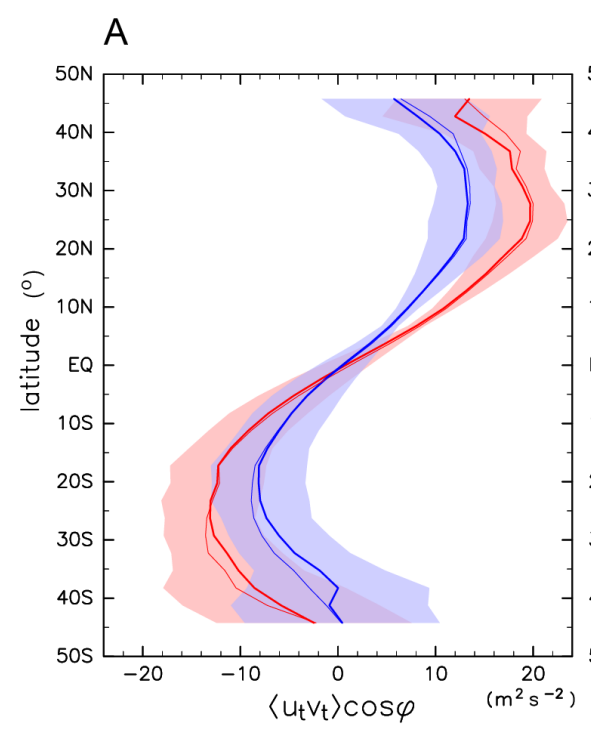

B $\quad$ C
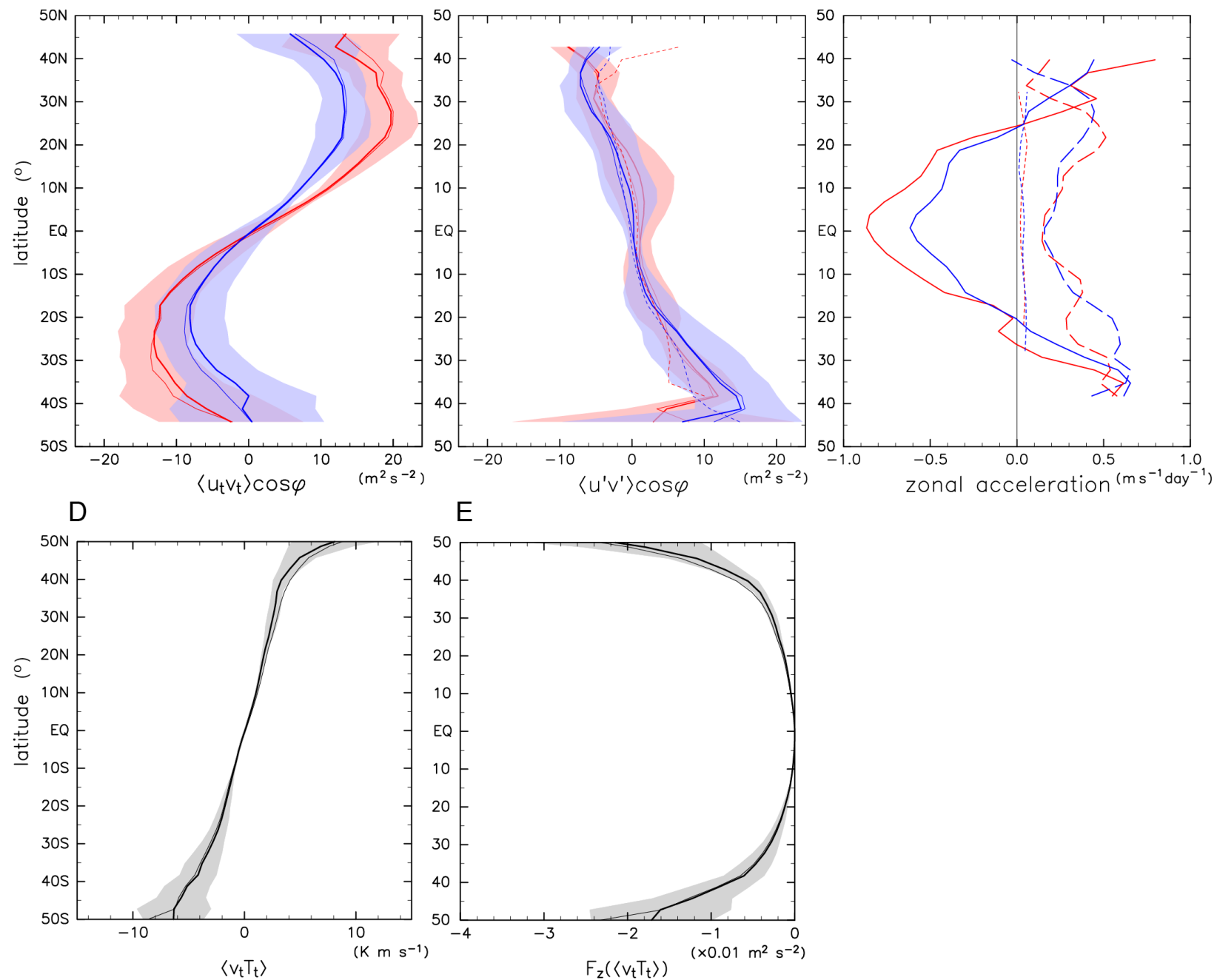

$\mathrm{E}$

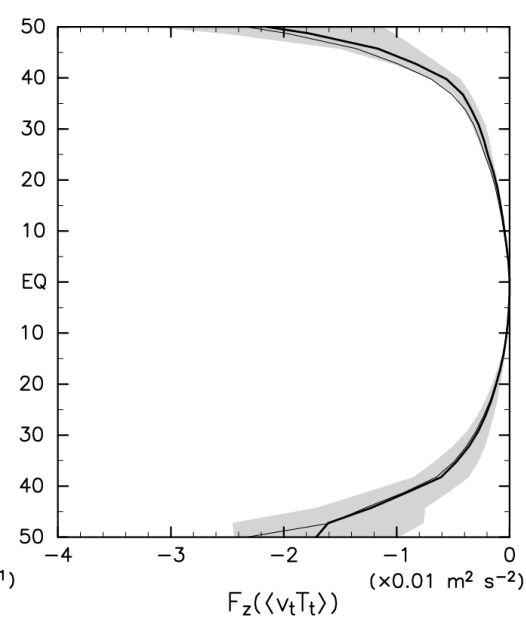

Fig. 2. Meridional AM flux and its divergence due to thermal tide and transient disturbances at the cloud top, and tidal heat flux. (A) Mean tidal flux (red: from 283-nm images; blue: $365-\mathrm{nm}$ ) obtained by averaging $\left\langle u_{t} v_{t}\right\rangle \cos \varphi$ for the second to fifth subperiods (thick solid curves). The first subperiod was excluded because the local time coverage was too narrow to estimate $u_{0}$. (A,B,D,E) Shading shows the standard deviation among subperiods. Thin solid curves are similar to thick solid curves but obtained without any subperiod division. (B) As in A but for transient disturbances, $\overline{\left\langle u^{\prime} v^{\prime}\right\rangle} \cos \varphi$, from the five subperiods. Biases arising from the error covariance between $u^{\prime}$ and $v^{\prime}$ have been subtracted. Dotted curves: as the thin solid curves but from daily-mean winds $\left(u^{\#}, v^{\#}\right)$. (C) Zonal acceleration by the meridional AM flux convergence by thermal tides $\left(-\frac{\partial\left\langle u_{t} v_{t}\right\rangle \cos ^{2} \varphi}{a \cos ^{2} \varphi \partial \varphi}\right.$; solid curves) and transient disturbances ( $-\frac{\partial\langle u \prime v \prime\rangle \cos ^{2} \varphi}{a \cos ^{2} \varphi \partial \varphi}$; dashed curves) based on the thin solid curves in A and B. Dotted curves: same but for Rossby waves obtained from the co-spectra after a 1:2:1 smoothing with latitude. Colors are as in A. (D) Mean tidal heat flux by diurnal tides obtained by averaging $\left\langle v_{t} T_{t}\right\rangle_{1}$ for the five subperiods with $v_{t}$ at $365 \mathrm{~nm}$ (thick solid curve). (E) As in (D) but for $f_{z T 1} \equiv \zeta_{a} S^{-1}\left\langle v_{t} T_{t}\right\rangle_{1} \cos \varphi$ (using absolute vorticity $\zeta_{a}$ in Fig. S3B and the stability $S=8 \times 10^{-3} \mathrm{~K} \mathrm{~m}^{-1}$ ). 

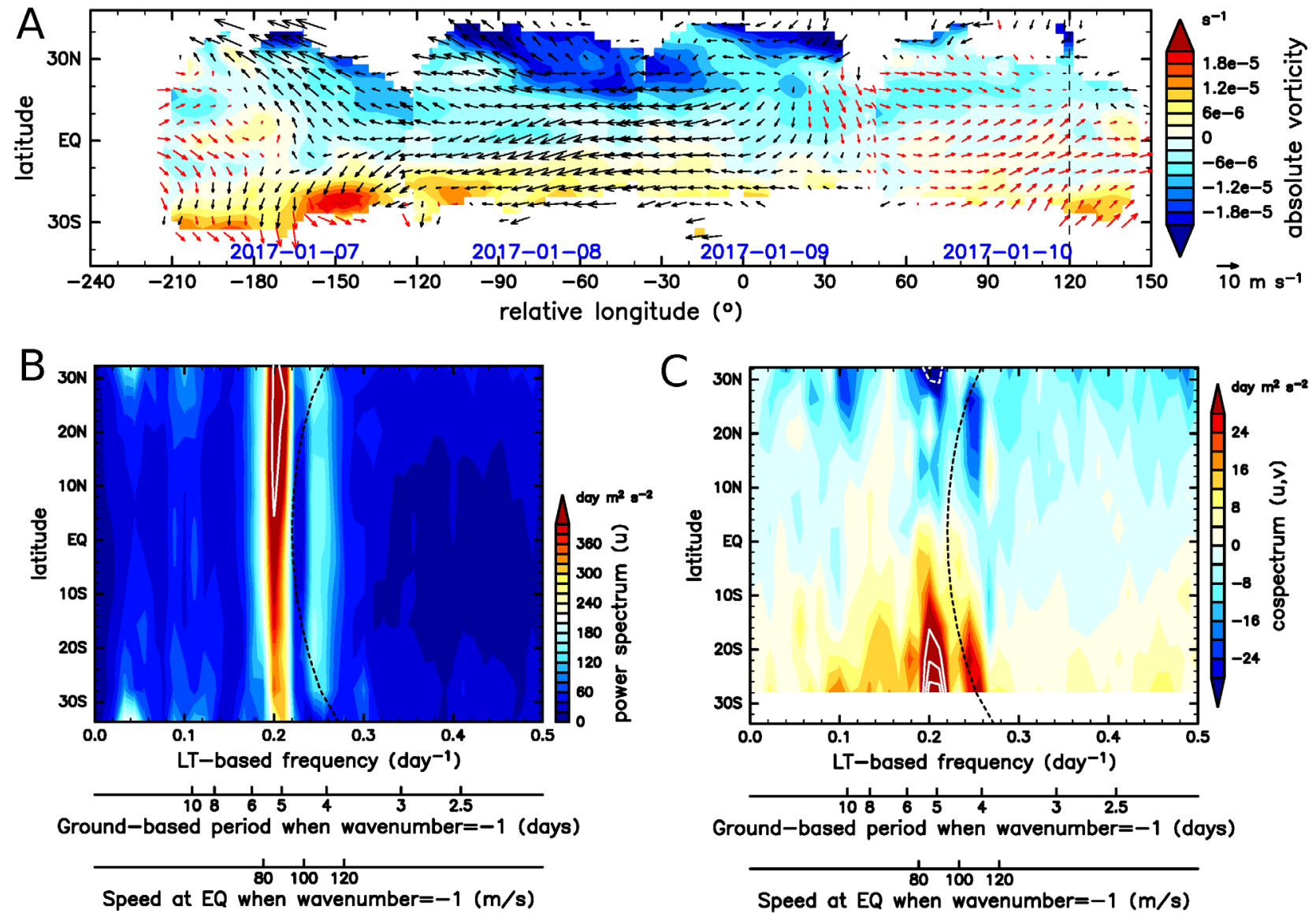

Fig. 3. Example wind disturbances (365-nm) and corresponding spectra. (A) daily-mean transient winds $\left(u^{\#}, v^{\#}\right)$ (arrows; red if $u^{\#}>0$ ) and absolute vorticity (color shading) for the period 2017 Jan $7-10$, overlaid by shifting $90^{\circ}$ per day. (B) power spectra of $u^{\#}$ obtained from the second to the fourth subperiods (color shading; the white contour is at $500 \mathrm{~m}^{2} \mathrm{~s}^{-2}$ day). Frequency is for observers at fixed local time (LT), $\tau_{L}$; the corresponding ground-based period (days) and zonal propagation speed at the equator (EQ) when the disturbances have zonal wavenumber 1 and move westwards (negative wavenumber) are shown on the abscissa. (C) as in (B) but for co-spectra between $u^{\#}$ and $v^{\#}$ (white contours are at an interval of $20 \mathrm{~m}^{2} \mathrm{~s}^{-2}$ day). 
A $(\mathrm{km})$

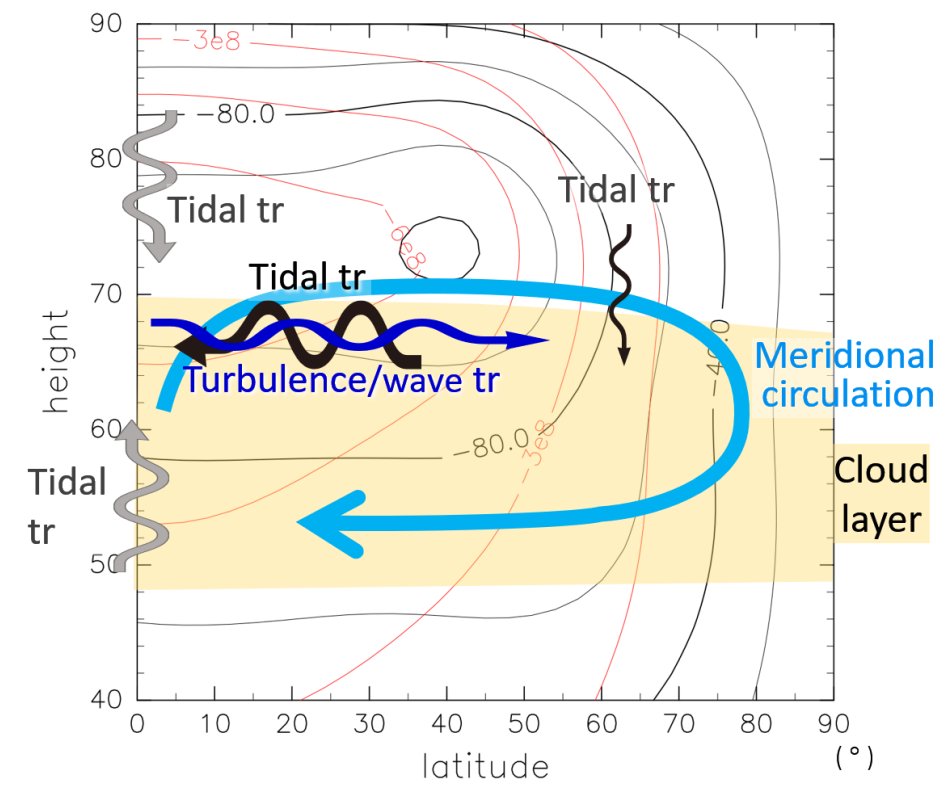

B $(\mathrm{km})$

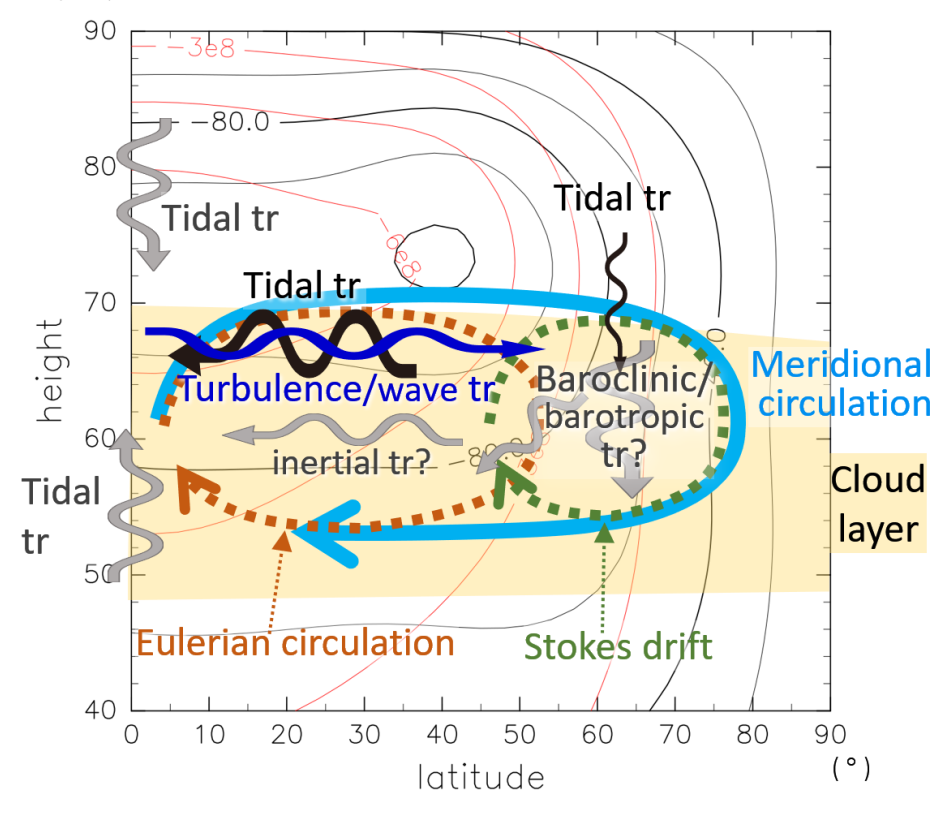

Fig. 4. Schematic illustration of our suggested AM balance in the cloud layer of Venus. A: processes quantified or estimated in this study; B: Same as A with our suggested interpretation overlain. Wavy arrows indicate eddy transport of SR's negative AM; those labelled with "tr" represent transport processes we quantified (black, blue) or estimated (gray), and those with "tr?" (in B) represent suggested ones. Their widths crudely represent their relative contribution to the AM transport. Cyan curved arrow shows the mean meridional circulation that acts to homogenize AM. The dotted curved arrows are expected contributions to the circulation (16). The background shows the cloud layer (pale orange shading), idealized distributions of $\langle u\rangle$ (black contours; $\mathrm{m} \mathrm{s}^{-1}$ ), and $M$ (red contours; $\mathrm{m}^{2} \mathrm{~s}^{-1}$ ). 


\section{Science MIAAAS}

\section{Supplementary Materials for}

How waves and turbulence maintain the super-rotation of Venus' atmosphere

Takeshi Horinouchi, Yoshi-Yuki Hayashi, Shigeto Watanabe, Manabu Yamada, Atsushi Yamazaki, Toru Kouyama, Makoto Taguchi, Tetsuya Fukuhara, Masahiro Takagi, Kazunori Ogohara, Shin-ya Murakami, Javier Peralta, Sanjay S. Limaye, Takeshi Imamura, Masato Nakamura, Takao M. Sato, Takehiko Satoh

Correspondence to: horinout @ ees.hokudai.ac.jp

\section{This PDF file includes:}

Materials and Methods

Supplementary Text

Figs. S1 to S11

Table S1

References (34-56) 


\section{Table of contents}

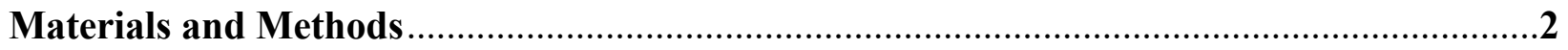

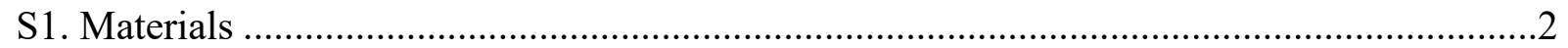

S2. Symbols and definitions...................................................................................

S3. A model of heat-induced meridional circulation and idealized zonal wind distribution......4

S4. Wind estimation from Akatsuki data ...................................................................6

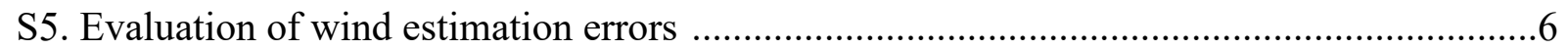

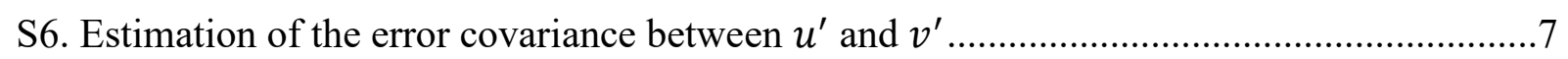

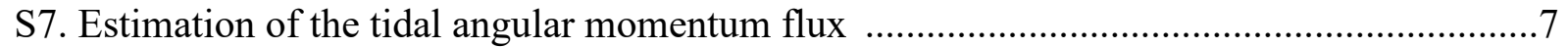

S8. On the estimation of the time mean values used to extract transient disturbances .............9

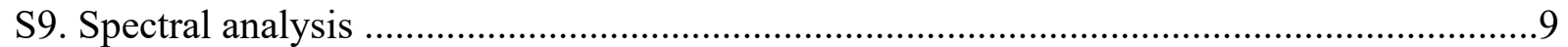

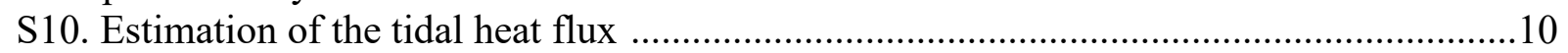

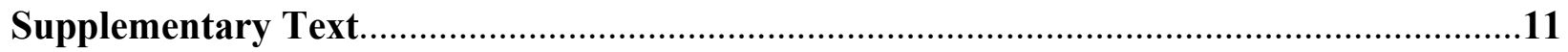

S11. Meridional circulation consistent with preceding estimates of net radiative heating ......11

S12. AM tendency by the meridional circulation, roles of hydrodynamic instabilities, and

Eulerian vs eddy-induced (Stokes drift) circulations

S13. Consistency of the heat-induced meridional circulation model with satellite-indicated

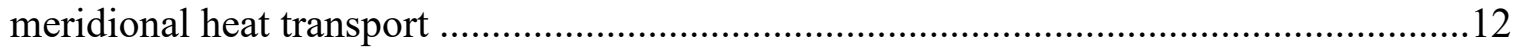

S14. Relative importance of the two terms in the meridional component of the EP flux ........12

S15. Order estimation of the vertical component of the EP flux ........................................12

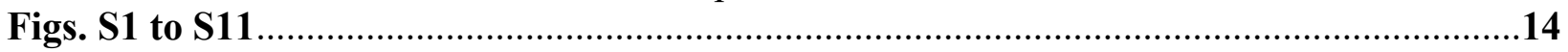

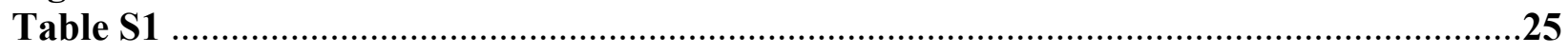

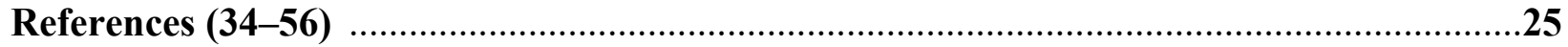

\section{Materials and Methods}

\section{S1. Materials}

We use data from the Venus orbiter Akatsuki (34,35), mostly the Level 3 product of the UV radiance at 283 and $365 \mathrm{~nm}$ obtained from Akatsuki/UVI (31). The particular version we used is the internal release version v20180601 up to imaging until Mar 2018 and v20190301 for the rest, but the difference among the versions are very small and do not affect the results of cloud tracking (the results are identical). We also used the version v20190201 Level 3C product of the brightness temperature from Akatsuki/LIR (32).

We also examined previously-derived (36) winds at the lower-to-middle cloud layer from the near-infrared data from Akatsuki/IR2 (33). However, we could not estimate the AM flux reliably, presumably because the observation period was short and the tidal amplitude is small there (37), so the results are not presented.

We also used the downward solar flux based on Pioneer Venus sounder (38), which is compiled in the Venus International Reference Atmosphere (VIRA) (39). The global mean profile as a function of altitude, $F(z)$, was taken from thier Figure 6-13 and listed in Table S1. 


\section{S2. Symbols and definitions}

For three-dimensional vector, the components are, in order, zonal (positive when eastward), meridional (positive when northward), and vertical (positive when upward).

\section{List of symbols}

$t$ : time.

$\tau_{L}$ : local time defined by assigning $0-24 \mathrm{~h}$ to longitude relative to the anti-solar point. ( $\tau_{L}$ decreases with longitude, since Venus rotates westward. $\tau_{L}=12 \mathrm{~h}$ at the sub-solar longitude.) $\varphi$ : latitude (in radian).

$z$ : vertical coordinate (log-pressure height as is conventional in meteorology).

$M$ : angular momentum with respect to the rotational axis per unit mass.

$u$ : zonal wind (eastward velocity component).

$v$ : meridional wind (northward velocity component).

$w$ : vertical wind (upward velocity component along the log-pressure height $z$ ).

$\boldsymbol{F} \equiv\left(0, F_{y}, F_{z}\right)$ : the Eliassen-Palm (EP) flux.

$\boldsymbol{v}^{r} \equiv\left(0, v^{r}, w^{r}\right)$ : The residual circulation (40), which are the zonal-means (means over eastwest circles) of $v$ and $w$, respectively, added with corrections to account for the Stokes drift to approximate the mean meridional circulation; the Stokes drift terms makes this velocity reflect air-mass transport better than without them. By definition, the residual circulation is zonally uniform.

$\Omega$ : the angular velocity of the rotation of the solid planet $(\Omega=-2 \pi /(243$ days $)$; negatively valued, since Venus rotates westward).

$a$ : the planetary radius (set to $6.12 \times 10^{6} \mathrm{~m}$ considering the cloud top height at around $70 \mathrm{~km}$ ). $\rho_{0}$ : normalized pressure multiplied with a constant reference density (a conventional quantity used in the log-pressure coordinate).

$S$ : the static stability in terms of temperature gradient (vertical temperature gradient plus adiabatic lapse rate, if the log-pressure scale height is set from the local temperature). $\zeta_{a}$ : the vertical component of absolute vorticity.

\section{Definitions of the means and deviations}

$\langle x\rangle$ : zonal mean (average along longitude) of $x$. Quantities that are zonal means by definition ( $M$, $\left.\boldsymbol{v}^{r}, \boldsymbol{F}\right)$ are expressed without angular brackets.

$x^{*} \equiv x-\langle x\rangle$ : deviation from the zonal mean.

$\bar{x}$ : time mean of $x$ for fixed $\tau_{L}$ and $\varphi$.

$x^{\prime \prime} \equiv x-\bar{x}:$ deviation from the time mean. 
$\tilde{x}$ : temporal running mean of $x$ over \pm 15 days for fixed $\tau_{\mathrm{L}}$ and $\varphi$.

$x^{\prime} \equiv x-\tilde{x}$ : deviation from the temporal running mean.

$x^{\#}$ : daily mean of a quantity $x$ derived by cloud tracking (thus $x$ is $u$ or $v$ ) over a universal-time based calendar day for fixed $\tau_{\mathrm{L}}$ and $\varphi$.

\section{Some relationships}

Angular momentum:

$$
M=a\langle u\rangle \cos \varphi+a^{2} \Omega \cos ^{2} \varphi
$$

EP flux:

$$
\begin{aligned}
& F_{y}=\rho_{0} a\left(f_{y v}+f_{y T}\right), \\
& F_{z}=\rho_{0} a\left(f_{z w}+f_{z T}\right),
\end{aligned}
$$

where

$$
\begin{gathered}
f_{y v} \equiv-\left\langle u^{*} v^{*}\right\rangle \cos \varphi \\
f_{y T} \equiv \frac{\partial\langle u\rangle}{\partial z} S^{-1}\left\langle v^{*} T^{*}\right\rangle \cos \varphi, \\
f_{z w} \equiv-\left\langle u^{*} w^{*}\right\rangle \cos \varphi \\
f_{z T} \equiv \zeta_{a} S^{-1}\left\langle v^{*} T^{*}\right\rangle \cos \varphi .
\end{gathered}
$$

See (40) for further information on the transformed Eulerian mean equations.

\section{S3. A model of heat-induced meridional circulation and idealized zonal wind distribution}

A rough estimation of the mean Lagrangian (i.e., following air-pacel motions) meridional circulation $\boldsymbol{v}^{r}$ is derived from idealized diabatic heating distribution. We assume that longwave heating up to $100 \mathrm{~km}$ and convective heating in the lower cloud region have much smaller meridional contrast than the heating by the absorbed solar radiation. Therefore, we assume that the meridional diabatic heating structure is determined solely by that of the solar-heating, which is assumed to be proportional simply to $\cos \varphi$ (to elaborate the meridional structure does not provide a big difference). Then, the diabatic heating per unit mass is expressed as

$$
Q=h(\varphi) \frac{\partial F(z)}{\rho \partial z}
$$

where $h(\varphi) \equiv \frac{4}{\pi} \cos \varphi-1$, and $\rho$ is density. Here, the term -1 in $h$ corresponds to the meridionally uniform longwave/convective heating to make the global-mean het heating vanish, and $\frac{4}{\pi}$ is the factor to normalize the integration of $\cos \varphi=(1-\mu)^{\frac{1}{2}}$ by $\mu$.

The vertical profile of $F$ is set from Table $\mathbf{S} 1$ by using the cubic spline interpolation. Here, we set $F=0$ at $z=0$ (Table S1) to roughly incorporate the heating by the absorbed solar-flux at the ground nearly equally between 0 to $10 \mathrm{~km}$. The resultant $F(z)$ is shown as Fig. 1A.

We introduce the non-dimensional log-pressure height $\zeta \equiv-\ln p / p_{00}\left(p_{00}\right.$ is a constant pressure representing the surface pressure) and define the normalized log-pressure vertical velocity $w^{*} \equiv \frac{D \zeta}{D t}$. Since the scale height varies greatly with altitude on Venus, we define the log- 
pressure vertical velocity as $w \equiv H w^{*}$, where $H=\frac{R T}{g}$ depends on altitude ( $R$ is the gas constant and $g$ is the gravity acceleration), unlike the convention to use a global constant scale height (40). At steady state, if the horizontal temperature advection tendency is neglected, the residualmean vertical velocity normalized in this way $w^{* r}$ is related to $Q$ as

$$
H S w^{* r}=Q \quad(\mathrm{~S} 9)
$$

The stability in terms of the temperature gradient $S$ is expressed as

$$
S=\Gamma+\frac{\partial \mathrm{T}}{\partial z}
$$

where $\Gamma$ is the adiabatic lapse rate. If we introduce the normalized meridional velocity $v^{*} \equiv \frac{D \mu}{D t}$, it is related to the (ordinary) meridional velocity as $v=a(\cos \varphi)^{-1} v^{*}$. The normalized residual velocity components can be related though a kind of mass stream function $\chi$ :

$$
p v^{* r}=-\frac{\partial \chi}{\partial \zeta}, p w^{* r}=\frac{\partial \chi}{\partial \mu}
$$

Equations (S8) - (S11) were solved for $\chi$ (shown in Fig. 1) by using $F$ as explained above, as well as $T, g$, and $\Gamma$ available in VIRA (39). We imposed a minimum value of $S$ as $1 \times 10^{-3} \mathrm{~K} \mathrm{~m}^{-1}$. The resultant $\chi$ had a spiky peak at around $55 \mathrm{~km}$ owing to the low stability region, whose strength depends on the imposed minimum value. Since it is likely a spurious peak, we removed it by linearly interpolating $\chi$ between 50 and $60 \mathrm{~km}$, as shown by dashed parts of the curves in Fig. 1B, D.

Meridional heat flux per unit vertical increment is expressed as $a \cos \varphi s v^{r}=a^{2} s v^{* r}$, where $s$ is the static energy per unit mass,

$$
s=h+\Phi
$$

Here, $\Phi$ is geopotential, which can be obtained by vertically integrating $g$, and $h$ is the specific enthalpy. We computed $h^{\prime} \equiv h-h(z=0 \mathrm{~km})$ by the integration $\int\left(\frac{\partial h}{\partial T}\right)_{p} d T+\int\left(\frac{\partial h}{\partial p}\right)_{T} d p$ along the integral pass of the $p$ and $T$ profiles in VIRA by using $\left(\frac{\partial h}{\partial T}\right)_{p}=c_{p}$ and the relation

$$
\Gamma=\frac{g}{c_{p}}\left[1-\rho\left(\frac{\partial h}{\partial p}\right)_{T}\right]
$$

which was used in the original derivation of $\Gamma$ in VIRA. Actually, the $\Gamma$ data is available only up to $72 \mathrm{~km}$. Above it, $\left(\frac{\partial h}{\partial p}\right)_{T}$ was set to be zero as in the ideal gas, which is valid at the relatively low pressure above $70 \mathrm{~km}$. The result is shown in Fig. $\mathbf{S 1 .}$

For a rough estimation of zonal acceleration associated with meridional circulation, an idealized meridional structure of zonal wind was defined between 40 and $90 \mathrm{~km}$ by considering observations $(18,24,39,41)$. Its meridional structure is specified analytically at selected altitudes $z_{i}(i=1,2, \ldots, 5)$ as

$$
u=-u_{0 i}\left[1+a_{i} \tanh \frac{\varphi-a_{c i}}{a_{w i}}+b e^{-\left(\frac{\varphi-b_{c i}}{b_{w i}}\right)^{2}}\right] \cos \varphi
$$

where $z_{i}=40,53,63,73,90 \mathrm{~km}, u_{0 i}=50,70,90,110,40 \mathrm{~m} \mathrm{~s}^{-1}, a_{i}=0.6,0.6,0.3,0.25,0.15$, $a_{w i}=30^{\circ}, 30^{\circ}, 25^{\circ}, 20^{\circ}, 20^{\circ}, a_{c i}=60^{\circ}, 60^{\circ}, 50^{\circ}, 38^{\circ}, 35^{\circ}, b_{i}=0.3,0.3,0.25,0.2,0.1, b_{w i}=$ $25^{\circ}, 25^{\circ}, 25^{\circ}, 15^{\circ}, 15^{\circ}$, and $b_{c i}=70^{\circ}, 70^{\circ}, 55^{\circ}, 45^{\circ}, 45^{\circ}$. It was vertically interpolated by using the cubic spline interpolation. The idealized zonal wind and the corresponding AM thus obtained are shown in Figs. 1C and 4. Note that $u$ is maximized around $z \sim 73 \mathrm{~km}$, which is slightly 
above the cloud top. This setting is because the mean wind obtained from the 283 -nm images is faster than that from the $365-\mathrm{nm}$ images (24).

\section{S4. Wind estimation from Akatsuki data}

We used the cloud tracking method derived for Akatsuki $(21,22)$. The parameter setting is the same as in earlier studies (24). Our tracking provides wind data on a $3^{\circ} \times 3^{\circ}$ grid from each of the three consecutive 2-hourly images over 4 hours. Each wind vector represents cloud motion over a $\sim 10^{\circ} \times 10^{\circ}$ region, so there is over-sampling on the $3^{\circ} \times 3^{\circ}$ grid. Wind data to derive wind disturbances $\left(u^{\prime}\right.$ and $\left.v^{\prime}\right)$ were smoothed over $3 \times 3$ grid points. In this case, the wind data used represent horizontal winds over slightly greater regions.

The quality control of the cloud tracking data is the same as in a previous study (24), but the threshold of one of the parameters, the parameter $\varepsilon$ in the study (a measure of precision), was raised (loosened) to $<20 \mathrm{~m} \mathrm{~s}^{-1}$ from $<10 \mathrm{~m} \mathrm{~s}^{-1}$, in order to obtain greater number of vectors at mid latitude. Over the three-year analysis period, the cloud-top winds are obtained from 3,789 image triplets at $365 \mathrm{~nm}$. The number of wind vectors that passed the quality control is over 1.4 million in the control case in which the $\varepsilon$ threshold was set to $20 \mathrm{~m} \mathrm{~s}^{-1}$. Also, 3,793 image triplets at $283 \mathrm{~nm}$ were used to obtain 1.2 million wind vectors. The number of validated vectors at each latitudinal grid is shown Fig. S3A for the 365-nm case; the profile for the 283-nm case is similar.

\section{S5. Evaluation of wind estimation errors}

In previous publications $(24,36)$, the actual uncertainty of our cloud tracking results was unknown. It was derived a priori from the screening parameters.

In this study, however, we devised a method to evaluate the uncertainty arising from wind estimation errors directly from the results. We introduce a "Lagrangian wind difference" of wind components, $\Delta u \equiv u^{\prime}\left(\tau_{L}+\Delta \tau_{L}, \varphi+\Delta \varphi, t+\Delta t\right)-u^{\prime}\left(\tau_{L}, \varphi, t\right)$ and $\Delta v \equiv v^{\prime}\left(\tau_{L}+\Delta \tau_{L}, \varphi+\right.$ $\Delta \varphi, t+\Delta t)-v^{\prime}\left(\tau_{L}, \varphi, t\right)$, where $\Delta \tau_{L}$ and $\Delta \varphi$ are the LT (associated with longitude) and latitude increments corresponding to the advection by $\left[u\left(\tau_{L}, \varphi, t\right), v\left(\tau_{L}, \varphi, t\right)\right]$ (full wind including the background) over $\Delta t$. Therefore, $\Delta u=\Delta v=0$, if the measurement error is zero and $u^{\prime}$ and $v^{\prime}$ do not change over the advection. In other words, the wind change in this case is solely associated with the mean tidal motion included in $(\bar{u}, \bar{v})$, so non-zero $\Delta u$ and $\Delta v$ arise from measurement error and actual transient wind disturbances (except the tide).

The standard deviations of $\Delta u$ and $\Delta v$, denoted as $\sigma_{\Delta u}$ and $\sigma_{\Delta v}$, respectively, are therefore the square roots of the summations of the variances due to measurement error and the variances of the actual advective change of the wind disturbances. These values can be regarded to represent the uncertainty if the latter contribution is much smaller than the former. Let $\mathrm{E}\left((\Delta u)^{2}\right)$ be the ensemble mean over $\tau_{L}$ and $t$ for each grid point of $\varphi$, and let $\mathrm{v}_{v}(\Delta t)$ defined similarly from $\Delta v$. Measured $u^{\prime}$ can be regarded as the sum of its true value and the measurement uncertainties. If the errors at $t$ and $t+\Delta t$ are uncorrelated and that signal and error are uncorrelated, the expected value of $\mathrm{E}\left((\Delta u)^{2}\right)$ is $2 \sigma_{\epsilon_{u}}^{2}+2\left(1-r_{u^{\prime}}(\Delta t)\right) \sigma_{u^{\prime}}^{2}$, where $\sigma_{\epsilon_{u}}^{2}$ is the variance of error in zonal wind estimation, $\sigma_{u^{\prime}}^{2}$ is the true (physical) variance of $u^{\prime}$, and $r_{u^{\prime}}(\Delta t)$ is the auto-correlation of $u^{\prime}$ at the lag of $\Delta t$ along advection. Therefore, if $\sigma_{u^{\prime}}^{2}$ or $\Delta t$ is 
sufficiently small, $\sigma_{\Delta_{u}} \equiv \sqrt{\frac{\mathrm{E}\left((\Delta u)^{2}\right)}{2}} \sim \sigma_{\epsilon_{u}}$ (note that $r_{u^{\prime}}(\Delta t) \rightarrow 1$ when $\left.\Delta t \rightarrow 0\right)$. Also, $\sigma_{\Delta_{v}} \equiv$ $\sqrt{\frac{E\left((\Delta v)^{2}\right)}{2}} \sim \sigma_{\epsilon_{v}}$, if $\sigma_{v^{\prime}}^{2}$ or $\Delta t$ is sufficiently small.

Figure S4 shows $\sigma_{\Delta_{u}}$ and $\sigma_{\Delta_{v}}$ for $\Delta t=4$ and 8 hours. Because the difference between $\Delta t=$ 4 and 8 hours is actually small at latitudes lower than $40^{\circ}$, the above requirement is satisfied. Therefore, we use $\sigma_{\Delta_{u}}$ and $\sigma_{\Delta_{v}}$ for $\Delta t=4$ hours as our uncertainty estimation. When $\Delta t=4$ hour is used, there is one common image used in the estimations of winds at $t$ and $t+\Delta t$. In principle, that can influence the estimation, but its effect is limited.

A possible alternative definition of $\Delta u$ and $\Delta v$ could be to use the Lagrangian wind difference of the full winds $u$ and $v$ rather than $u^{\prime}$ and $v^{\prime}$. We tested this version and found that the variance increases. Since the increase must be due to the actual (physical) one owing to the neglection of tidal acceleration, we should rather use the above-mentioned definition with $u^{\prime}$ and $v^{\prime}$.

The uncertainties of the daily-mean transient wind disturbances $u^{\#}$ and $v^{\#}$ can be evaluated as follows. These daily mean winds are defined only when 3 or more $u^{\prime}$ (or $v^{\prime}$ ) values are available, and the typical number used in averaging is 5 . Therefore, the error variance should be approximately $1 / 5$ times. Therefore, the uncertainties of $u^{\#}$ and $v^{\#}$ can be taken to be $1 / \sqrt{5}$ times of $\sigma_{\epsilon_{u}}$ and $\sigma_{\epsilon_{v}}$, respectively.

\section{S6. Estimation of the error covariance between $u^{\prime}$ and $v^{\prime}$}

UV brightness features tends to be oriented slantwise in northwest-southeast (southwestnortheast) directions in the northern (southern) hemisphere (42). Though a high-pass filter is applied before tracking clouds (24), the overall slantwise feature creates greater ambiguity in the northwest-southeast directions than southwest-northeast directions in the northern hemisphere. It creates negative covariance in the errors in $u^{\prime}$ and $v^{\prime}$. Therefore, we evaluated the covariance from the data, and subtracted its effect from the AM flux.

Under the same assumption as in the previous subsection, the expected value of $\mathrm{E}(\Delta u \Delta v)$ is $2 \mathrm{C}\left(\epsilon_{u}, \epsilon_{v}\right)+2 \mathrm{C}\left(u^{\prime}, v^{\prime}\right)-\mathrm{C}\left(u^{\prime}, v^{\prime} ; \Delta t\right)-\mathrm{C}\left(u^{\prime}, v^{\prime} ;-\Delta t\right) \cong 2 \mathrm{C}\left(\epsilon_{u}, \epsilon_{v}\right)-\mathrm{C}\left(u^{\prime}, \frac{\partial^{2} v^{\prime}}{\partial t^{2}}\right) \Delta t^{2}$, where $\mathrm{C}(x, y)$ is the covariance of $x$ and $y$ with no time lag, and $\mathrm{C}(x, y ; \Delta t)$ is the covariance of $x$ and $y$ with the time lag of $\Delta t$. The last derivation is from the Taylor expansion (the last term can also be written as $\left.-\mathrm{C}\left(\frac{\partial^{2} u^{\prime}}{\partial t^{2}}, v^{\prime}\right) \Delta t^{2}\right)$. The result is shown in Fig. S5. Because the difference between the cases with $\Delta t=4$ hours and 8 hours is small, we can regard that $\frac{1}{2} \mathrm{E}(\Delta u \Delta v)$ at $\Delta t=4$ hours as the estimation of error covariance. This covariance was obtained for each subperiod and subtracted from the mean $u^{\prime} v^{\prime}$ to draw solid lines in Fig. 2B.

Following the argument in the previous subsection, the error covariance between $u^{\#}$ and $v^{\#}$ (daily-means) was supposed to be $1 / 5$ of the error covariance between $u^{\prime}$ and $v^{\prime}$, so it was set to $\frac{1}{10} \mathrm{E}(\Delta u \Delta v)$ at $\Delta t=4$ hours. It is actually quite small, so the subtraction affected the results (Fig. 2B) little.

\section{S7. Estimation of the tidal angular momentum flux}

The temporal mean winds obtained from observations $(\bar{u}, \bar{v})$ consists of the zonal and temporal mean winds and the tidal winds: $(\bar{u}, \bar{v})=\left(u_{0}+u_{t}, v_{0}+v_{t}\right)$. In order to derive the 
tidal AM flux using $\left(u_{t}, v_{t}\right)$, we need to separate $\left(u_{0}, v_{0}\right)$ from $(\bar{u}, \bar{v})$. However, the cloud-top wind is available only over the dayside, so a true separation is never possible. Therefore, we utilize a priori knowledge and evaluate its validity from conceivable ranges of $\left(u_{0}, v_{0}\right)$, as described below.

Observational studies of cloud-top temperature by thermal infrared $(29,43)$, which covers both dayside and nightside, showed that the temperature variation associated with the thermal tide has the greatest amplitude at the zonal wavenumber-2 (wavenumber-1) component at latitudes lower (higher) than $\sim 40^{\circ}$, which is the case in a recent modeling study (44). This modeling study also showed that the ratio between the zonal wavenumber- 1 and wavenumber- 2 components are similar between temperature and zonal wind, as expected from the theoretical equipartitioning of kinetic and potential energies in internal gravity waves. These studies indicate that not only temperature but also zonal-wind disturbances associated with the tide are dominated by the zonal wavenumber -2 component at latitudes lower than $30^{\circ}$, where the amplitude of wavenumber-1 component is less than one-third of the wavenumber- 2 component (29). Therefore, we can expect that the momentum flux can be estimated reasonably well from our observations limited to dayside at latitudes lower than $30^{\circ}$. We discuss this further below.

In our primary calculation used to create Fig. $2 \mathbf{A}$, we simply set $v_{0}=0$. In addition, we confirmed that using $v_{0}$ on $\sim 1 \mathrm{~m} \mathrm{~s}^{-1}$, as suggested in the subsections $\mathbf{S 1 1}$ and $\mathbf{S 1 2}$, changes Fig. 2A only slightly.

An elaborate estimation is needed to constrain $u_{0}$ to derive $u_{t}$ appropriately. Because of the dominance of the wavenumber- 2 component at low latitude, we estimated $u_{0}$ by fitting the wavenumber-2 sinusoidal curve. Figure S9 and $\mathbf{S 1 0}(\mathbf{A}-\mathbf{H})$ shows $\bar{u}$ at various latitudes obtained from cloud-tracking winds over the three years. The red dashed curves show the wavenumber-2 fit. The fitting results appear reasonable even at mid-latitude, because the wavenumber- 1 tidal zonal wind changes sign at around the sub-solar longitude. The zonal mean wind $u_{0}$ obtained from this fitting is shown as constant offsets to the sinusoidal curve. These $u_{0}$ values are used to estimate the AM flux in Fig. 2A, which is reproduced in Figs. S9I and S10I.

We also tested fitting by using both the wavenumber- 1 and wavenumber- 2 sinusoidal curves simultaneously. However, the results were unrealistic, giving $u_{0}$ frequently outside the maximum and the minimum of $\bar{u}$. This failure is presumably because of the shortness of the local-time coverage. An earlier study (7) used this two-wave fitting to define both $u_{0}$ and $v_{0}$.

An alternative definition of $u_{0}$ was tried by setting it to the center of the maximum and the minimum of $\bar{u}$ along LT. The AM flux obtained this way is similar to the one obtained by the wavenumber-2 fitting (Figs. S9-S10).

We also tried to constrain $u_{0}$ by range. Fig. $\mathbf{S 9}$ and $\mathbf{S 1 0}$ show their estimates obtained by the wavenumber- 2 fitting plus/minus the $30 \%$ of the wavenumber- 2 amplitude from the fitting. Because of the dominance of the wavenumber- 2 component, it is unlikely that true $u_{0}$ is outside the range at latitudes lower than $30^{\circ}$. Especially, the slower $u_{0}-$ limit case is unrealistically slow, because the mean-wind curves capture local wind-speed minima. Even in this case, the $\left\langle u_{t} v_{t}\right\rangle \cos \varphi$ curve (Figs. S9I and S10I) exhibit positive gradient at the equator.

In order to regard the curves in Fig. $\mathbf{2 A}$ as the AM flux, we need a further assumption. Because the flux is derived by taking the zonal mean only over the dayside, it is not the true zonal mean over the entire latitudinal circles. However, the dominance of the zonal wavenumber-2 component indicates that the estimation only over dayside provides reasonable estimation at low latitude. In a recent modeling study (45), the relative amplitude of the wavenumber- 1 component to the wavenumber- 2 component is greater than that is observed 
$(29,43)$. Despite the bias, the study showed that the computed meridional fluxes are similar between the true zonal mean and the partial zonal mean over dayside. Therefore, the bias caused by the imperfect LT coverage of $u_{t}$ and $v_{t}$ should be limited.

From these investigations, we conclude that the estimates in Fig. $2 \mathbf{A}$ is adequate at latitudes lower than $30^{\circ}$, and the sign and the order of magnitude of the tidal SR acceleration at low latitude shown in Fig. 2C is robust. However, the tidal AM flux and its convergence at higher latitudes may be inaccurate.

\section{S8. On the estimation of the time mean values used to extract transient disturbances}

We used $\left(u^{\prime}, v^{\prime}\right)$, where 31-day running-mean winds are subtracted. One can alternatively use $\left(u^{\prime \prime}, v^{\prime \prime}\right) \equiv(u-\bar{u}, v-\bar{v})$, where $(\bar{u}, \bar{v})$ are simple time means (for fixed $\tau_{\mathrm{L}}$ and $\varphi$ ) over each subperiod. This choice would be conceptually simpler because $(u, v)=\left(\bar{u}+u^{\prime \prime}, \bar{v}+v^{\prime \prime}\right)=$ $\left(u_{0}+u_{t}+u^{\prime \prime}, v_{0}+v_{t}+v^{\prime \prime}\right)$. However, it is not practically cleaner, since $\left(u^{\prime \prime}, v^{\prime \prime}\right)$ include not only transient spatial variations such as waves and turbulence but also slow variations of mean winds to some extent. Therefore, we used $\left(u^{\prime}, v^{\prime}\right)$ to exclude the latter. However, we confirmed that the AM flux obtained by using $\left(u^{\prime \prime}, v^{\prime \prime}\right)$ are similar to that derived from $\left(u^{\prime}, v^{\prime}\right)$.

\section{S9. Spectral analysis}

We computed frequency spectra with time, $t$, of daily-mean perturbation winds $u^{\#}\left(\tau_{L}, \varphi\right.$, $t)$, and $v^{\#}\left(\tau_{L}, \varphi, t\right)$ from the second to the fifth subperiods. Before computing the spectra, winds are binned over 3 grid points with longitude and 2 grid points with latitude. Since the data have gaps with time, we compared the Lomb-Scargle method (46) and the discrete Fourier transform (DFT) by using the Fast Fourier Transform (FFT), and we confirmed that the results are similar between the two methods (e.g., Fig. S8A), if we process data as in what follows. In our actual analysis, we use DFT (Fig. 2 and Fig. S8B-D).

The time stamp of our daily-mean winds is based on the average of the observational times in each day, so it is unequal. The Lomb-Scargle spectra were computed directly with the unequal times. As for the DFT, however, it is assumed that the time sampling is equal. DFT also requires that the time sequence has no data gap. In our computation, data gaps up to two days are interpolated, and DFT was conducted only when 45-day or longer time sequence is secured. The DFT was applied by using 90-day time windows for each subperiod, with zero-padding after the consecutive time sequences. The Lomb-Scargle periodogram was also computed with the same frequency increment as DFT, which is $1 / 90$ day $^{-1}$. The normalization of the Lomb-Scargle power spectra was based directly on Parseval's identity.

If the complex Fourier coefficient of a quantity $x\left(\tau_{L}, \varphi, t\right)$ is expressed as $X\left(\tau_{L}, \varphi, f\right)$, where $f$ is frequency, our power spectrum of $x$ is $c X\left(\tau_{L}, \varphi, f\right) X^{*}\left(\tau_{L}, \varphi, f\right)$ averaged with $\tau_{L}$ and further ensemble-averaged over subperiods for given $\varphi$ (actually, before the Fourier transform $x\left(\tau_{L}, \varphi, t\right)$ was binned over three longitudinal $\left(\tau_{L}\right)$ grid points and two $\varphi$ grid points having the $3^{\circ} \times 3^{\circ}$ grid spacing). Here, $c$ is the factor to make the power spectrum integrated over $f$ (up to the Nyquist frequency) is equal to the variance, and asterisk indicates complex conjugate. The co-spectra and the quadrature-spectra (the imaginary part of cross-spectrum) between $x\left(\tau_{L}, \varphi, t\right)$ and $y\left(\tau_{L}, \varphi, t\right)$ are the real and the imaginary parts of their cross spectrum, which is $c X\left(\tau_{L}, \varphi, f\right) Y^{*}\left(\tau_{L}, \varphi, f\right)$ averaged in the same way. The squared coherency, which represents correlation for each frequency, is the ratio between the squared absolute values of the cross spectrum and the power spectra of $x$ and $y$. 
The power spectra shown in Figs. $\mathbf{2}$ and $\mathbf{S 8}$ were computed from the four subperiods from the second to the fifth; the first subperiod was not used because its coverage was limited. The degree of freedom in each subperiod depends on autocorrelation along $\tau_{L}$ for each frequency. As for the zonal-wavenumber-1 wave, the degree of freedom along $\tau_{L}$ at each time should be nearly 1 , since the $\tau_{L}$ coverage is limited (24). However, in each subperiod, the $\tau_{L}$ coverage shifts gradually, and there is little overwrap in the $t$ range between the early "morning" $\tau_{L}$ and late "afternoon" $\tau_{L}$ (24). Therefore, the degree of freedom should be at least 2 for each of the four subperiods. In total, the spectra can be regarded to have 8 or more degree of freedom (i.e., 8 or more independent spectra are used to create the figure).

\section{S10. Estimation of the tidal heat flux}

Meridional tidal heat flux was estimated by using $v_{t}$ from the cloud tracking described above and the cloud-top brightness temperature data obtained by the thermal-infrared camera LIR onboard Akatsuki. LIR observes thermal infrared at 8-12 $\mu \mathrm{m}$ (47). When the satellite zenith angle is low (i.e., for the observation of around the sub-satellite point), the LIR measurement is sensitive to the temperature at $\sim 4 \mathrm{~km}$ below the cloud top to which UVI measurement is sensitive (48). Therefore, we used data obtained at high satellite zenith angles at which the slantwise path increases the representative altitude. The mean LIR brightness temperature is decreased by $\sim 12$ $\mathrm{K}$ at $70^{\circ}$ than at $0^{\circ}$, indicating higher representative altitude by $\sim 4 \mathrm{~km}$. We derived the tidal temperature field $T_{t}$ from the LIR brightness temperature observed when the satellite zenith angle is between $50^{\circ}$ and $80^{\circ}$. The overall temperature dependence on the zenith angle was corrected before deriving the mean temperature. The result obtained from the time mean over the entire three-year observational period is shown in Fig. S11A.

In Fig. 2D,E, the result is shown for the wavenumber-1 component, the diurnal tide. The diurnal component of $T_{t}$ was obtained simply from the Fourier transform with LT (see Fig. S11B for the three-year mean case). $v_{t}$, which is available only on the dayside, was obtained by fitting the wavenumber-1 sinusoidal curve at each latitude (Fig. S11C).

An alternative derivation was tested to use only the dayside results without wavenumber decomposition/fitting. The heat flux obtained in this way was smaller than that in Fig. 2D; around $1 / 2$ at around $50^{\circ} \mathrm{S}$ and $50^{\circ} \mathrm{N}$ mid-latitude and nearly zero at low latitude. This result is qualitatively expected, since the thermal tide includes semidiurnal (zonal-wavenumber 2) contributions; in the gravity-wave mode that dominate the semidiurnal tide, the meridional heat flux is expected to be reversed from that of Rossby waves, according to the inertia-gravity wave theory (40).

The near-cloud-top brightness temperature obtained by LIR is not necessarily the temperature at a pressure level. This is also the case for $v_{t}$, but because of thermal stratification, the horizontal structure of temperature is expected to be more sensitive to the altitude sampling than that of tidal winds. It may appear that the representative altitude for the LIR brightness temperature is more like an isentropic surface than an isobaric surface. However, according to the linear wave theory, the temperature disturbance is exactly the same in these two cases if the background vertical temperature profile is iso-thermal. Around the cloud top level, the background temperature decreases with altitude, so the isentropic tidal amplitude should be larger than that of the isobaric tidal amplitude, but the former is less than twice the latter, and the phase is identical. Therefore, the results shown in Fig. 2D,E should is expected be valid within a factor of $\sim 2$ in this respect. A further ambiguity arises from the partial zonal coverage of $v_{t}$ as described above, but we expect that it is not large (45). 


\section{Supplementary Text}

\section{S11. Meridional circulation velocities in the heat-induced meridional circulation model}

Our meridional circulation model (described in subsection S3) is simpler than but consistent with the preceding studies on the net radiative heating in the cloud layer (49-51). The net radiative effect is heating at low latitude and cooling at high latitude, implying thermally induced circulation around the cloud layer. Since the estimated net radiative heating at around the cloud top at low latitudes, $Q$, is around $2 \mathrm{~K} \mathrm{day}^{-1}$, and $S \sim 8 \times 10^{-3} \mathrm{~K} \mathrm{~m}^{-1}$, it is suggested that $w^{r} \sim Q / S \sim 0.3 \mathrm{~cm} \mathrm{~s}^{-1}$ as has been suggested (49). In this case, it takes about 40 days to ascend by $10 \mathrm{~km}\left(\sim 2 H\right.$, where $H$ is the scale height). Since the derived $w^{r}$ around the cloud top does

not vary strongly over a scale height, the continuity equation $\frac{\partial v^{r} \cos \varphi}{a \cos \varphi \partial \varphi}+\frac{\partial w^{r}}{\partial z}-\frac{w^{r}}{H}=0$ suggests that some substantial portion of vertical mass flux should be diverted to poleward within some scale heights. As a rough estimation, if it takes 40 days to travel meridionally by $45^{\circ}$, its mean meridional speed is $1.4 \mathrm{~m} \mathrm{~s}^{-1}$. Therefore, $v^{r}$ is likely $\sim 1 \mathrm{~m} \mathrm{~s}^{-1}$. This is one order of magnitude smaller than the earlier estimations from the cloud tracking only over the dayside (52, 24), which is sometimes interpreted as a rough estimation of the zonal-mean meridional wind even now (2).

\section{S12. AM tendency by the meridional circulation, roles of hydrodynamic instabilities, and Eulerian vs eddy-induced (Stokes drift) circulations}

We discuss here the implications of the idealized model consisting of the meridional circulation derived by the method described in subsection $\mathbf{S 2}$ and the idealized wind distribution defined also there. In our derivation, $\boldsymbol{v}^{r}$ is obtained by assuming that the meridional contrast in the heating is attributed solely to that in the solar heating derived from observed downward shortwave flux compiled as VIRA $(39,53)$. This assumption should be valid to the first approximation, since the longwave heating has much smaller meridional contrast (49).

The idealized model is visualized in Fig. 1. The magnitudes of $v^{r}$ and $w^{r}$ are consistent with the estimation in subsection S11 ( $v^{r}$ in Fig. 1D has a local maximum of $1.9 \mathrm{~m} \mathrm{~s}^{-1}$ at 70 $\mathrm{km}$ ). The meridional circulation has an equatorward return flow below $\sim 60 \mathrm{~km}$ (Fig. 1C), since more than half of shortwave absorption occur in the cloud layer (Fig. 1A). This cloud-level circulation and the near-surface circulation are suggested by early studies $(11,12,17,55)$.

The model provides the advective zonal wind acceleration $-(a \cos \varphi)^{-1} \boldsymbol{v}^{r} \cdot \boldsymbol{\nabla} M$ as shown in Fig. 1C. The positive acceleration (to decelerate the westward SR) is between 60 to $73 \mathrm{~km}$ at low latitudes owing to the upwelling upgradient of $|M|$. Its peak is around $0.5 \mathrm{~m} \mathrm{~s}^{-1} \mathrm{day}^{-1}$. This value is sensitive to shear strength, but the result suggests that eddy AM convergence of $\sim 1$ $\mathrm{m} \mathrm{s}^{-1}$ day $^{-1}$ is needed to maintain the AM peak around the equatorial cloud top.

In Fig. 1C, negative (positive) advective acceleration exists above (below) $\sim 65 \mathrm{~km}$ at midto-high latitude. The advection reaching high latitude inevitably creates barotropic and baroclinic instabilities (and possibly inertial instability), so eddies arising from the instabilities should work to counteract the advective AM homogenization (19,20). In particular, baroclinic instability can transport AM downward, creating a vertically aligned deceleration and acceleration pair. If eddy AM transport were absent, the iso-lines of $M$ must coincide with the streamlines of $\boldsymbol{v}^{r}$ as schematically illustrated in Fig. S2A. Given the observed distribution of $M$, however, it is 
unlikely the case. Therefore, the eddy AM flux associated with baroclinic instability should act as suggested above, whose effect is as if part of the AM transport shortcuts the meridional circulation, causing the decrease and increase of $|M|$ along the meridional circulation (Fig. S2B).

The residual circulation, which approximates mean meridional Lagrangian circulation is generally different from the Eulerian-mean meridional circulation. The difference is due to the Stokes drift term parallel to the isolines of $\rho_{0} S^{-1}\left\langle v^{*} T^{*}\right\rangle \cos \varphi$, which is proportional to $f_{z T}$ (40). Therefore, baroclinic instability discussed above induces Stokes drift, while the Eulerian circulation is expected to be dominant at low latitudes, as in the Earth's atmosphere as schematically illustrated in Fig. 4B.

\section{S13. Consistency of the heat-induced meridional circulation model with satellite-indicated meridional heat transport}

It has been shown that net downward solar insolation is greater (smaller) than the outgoing longwave radiation at low latitudes (high latitudes) [(39), their Figure 6-4]. If the difference shown in the figure (shown for each $10^{\circ}$ latitudinal bin) is integrated from the equator, the total meridional heat flux peaks $40^{\circ}$ with the value of $3.6 \times 10^{15} \mathrm{~W} \mathrm{~s}^{-1}$. The heat flux must be provided by the meridional circulation of the atmosphere.

From subsection $\mathbf{S 2}$, vertically integrated heat flux between altitudes $z_{1}$ and $z_{2}$ is expressed as $E\left(z_{1} ; z_{2}\right) \equiv \int_{z_{1}}^{z_{2}} a^{2} s v^{* r} \rho d z$. For the idealized meridional circulation and $s$ in Fig. 1, $E(0 \mathrm{~km} ; 100 \mathrm{~km})$ was computed to be $3.5 \times 10^{15} \mathrm{~W} \mathrm{~s}^{-1}$, which is nearly equal to the above estimation. The agreement indicates that the idealized meridional circulation is not unrealistic. $40^{\circ}$ is close to $\varphi_{\max }=38.2^{\circ}$.

The heat flux can be computed between arbitrary altitudes, but in order for it to be useful, $z_{1}$ and $z_{2}$ should be taken so that the net meridional mass flux between them is nearly zero. Figure $1 B$ indicates that $\chi_{\max }(48 \mathrm{~km}) \sim \chi_{\max }(74 \mathrm{~km})$, so the net meridional mass flux between them are nearly zero (in this idealized meridional circulation). The derived $E(48 \mathrm{~km} ; 74 \mathrm{~km})$ value is $2.0 \times 10^{15} \mathrm{~W} \mathrm{~s}^{-1}$, which is about a half of the total heat transport.

\section{S14. Relative importance of the two terms in the meridional component of the EP flux}

The meridional component of the EP flux $F_{y}$, is proportional to $f_{y v}+f_{y T}$, where $f_{y v}=$ $-\left\langle u^{*} v^{*}\right\rangle \cos \varphi$ and $f_{y T}=\frac{\partial\langle u\rangle}{\partial z} S^{-1}\left\langle v^{*} T^{*}\right\rangle \cos \varphi=\left(\zeta_{a}^{-1}\right) f_{z T}$. The mean zonal wind shear $\left|\frac{\partial\langle u\rangle}{\partial z}\right|$ is likely smaller than $3 \mathrm{~m} \mathrm{~s}^{-1} \mathrm{~km}^{-1}$ from previous knowledge (41), so $\left|\zeta_{a}^{-1}\right|$ is less than 300 at $\sim 30^{\circ} \mathrm{N} / \mathrm{S}$ from Fig. S3B. Therefore, $\left|f_{y T}\right|$ by the diurnal tide is $\sim 1 \mathrm{~m}^{2} \mathrm{~s}^{2}$ or smaller from Fig. 2E, while $\left|f_{y v}\right|$ is $\sim 10 \mathrm{~m}^{2} \mathrm{~s}^{2}$. This order of magnitude estimation suggests that $F_{y}$ is dominated by $f_{y v}$ for the thermal tide. We could not have obtained reliable estimates of the heat flux for transient disturbances consistent among the subperiods, as mentioned in the text, but their order of magnitude we obtained was smaller than that of the thermal tide. Therefore, $F_{y}$ of the transient disturbances is likely dominated by $f_{y v}$ too.

\section{S15. Order estimation of the vertical component of the EP flux}

Direct estimation of the vertical component of the EP flux associated with tidal gravity waves is not available, since vertical wind measurement is not available. Therefore, we instead estimate its order of magnitude indirectly from their amplitude. Zonal wind amplitude of the 
thermal tide shown in Fig. S3C is $\sim 7 \mathrm{~m} \mathrm{~s}^{-1}$ at low latitude, which we denote as $U$. As a gravity wave, the wave energy per unit mass, $E$, is $\sim \frac{1}{2} U^{2}+$ potential energy, so $E \sim U^{2} \sim 50 \mathrm{~m}^{2} \mathrm{~s}^{-2}$. The vertical component of the EP flux, $F_{z}$, is approximately equal to $-c_{g z} \hat{c}^{-1} E$, where $c_{g z}$ is the vertical group velocity and $\hat{c}$ is the intrinsic zonal phase velocity (therefore $\hat{c} \sim 100 \mathrm{~m} \mathrm{~s}^{-1}$ ), if factors are adjusted so that $\frac{\partial F_{z}}{\partial z}$ represents zonal-wind acceleration. As for gravity wave, $c_{g z} \sim N k m^{-2}$, where $N$ is buoyancy frequency $\left(\sim 0.02 \mathrm{~s}^{-1}\right)$, and $k$ and $m$ are zonal and vertical wavenumbers, respectively. If we suppose the semidiurnal tide, the vertical wavelength is $\sim 20$ $\mathrm{km}$ theoretically (56) and from LIR observations (29), so $c_{g z} \sim 0.07 \mathrm{~m} \mathrm{~s}^{-1}$ and $F_{z} \sim 0.035$ $\mathrm{m}^{2} \mathrm{~s}^{-2}$. Therefore, if $F_{Z}$ diverges on the vertical scale of $Z \equiv 10 \mathrm{~km}$, $\left|\frac{\partial F_{z}}{\partial z}\right| \sim\left|F_{z}\right| Z^{-1} \sim 0.3 \mathrm{~m} \mathrm{~s}^{-1} \mathrm{day}^{-1}$. If we rather suppose the diurnal tide, the acceleration is smaller than the semidiurnal tide, since $c_{g z}$ is smaller. 


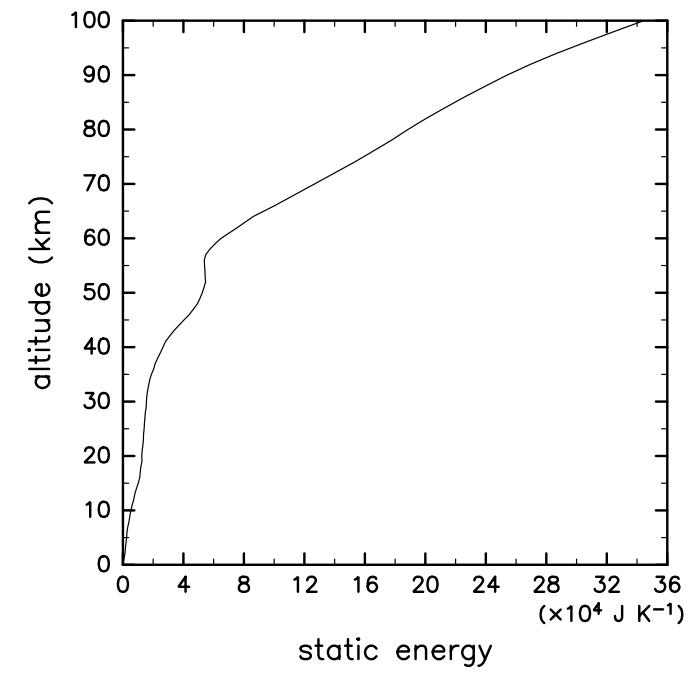

Fig. S1. A vertical distribution of static energy. It is obtained from the VIRA data (39), and is expressed as the difference from the surface value, $s^{\prime} \equiv s-s(z=0 \mathrm{~km})$. This profile was used to compute the meridional heat transport shown in subsection S13. Its theoretical basis is found in (54). 


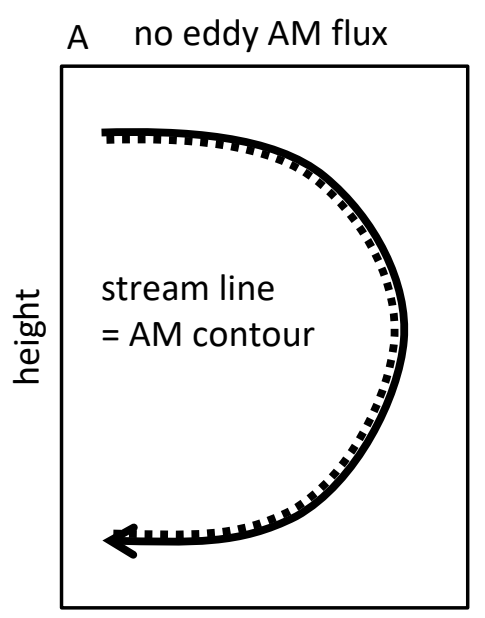

latitude

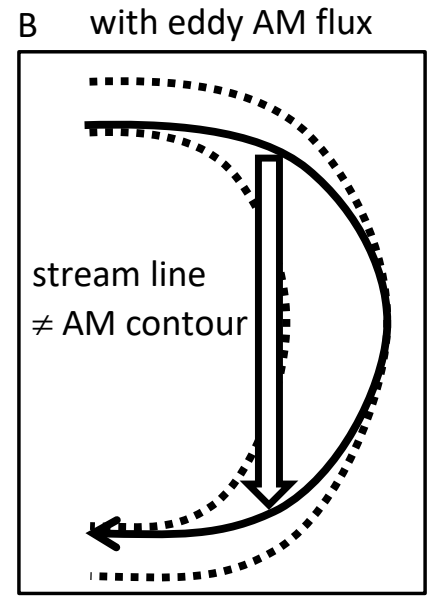

latitude

Fig S2. Schematic illustration of possible steady-state distribution of AM and meridional circulation at mid-to-high latitude (subsection S12). (A) When eddy AM transport is absent. In this case, the iso-lines of $M$ (dotted) must coincide with streamlines of mean Lagrangian meridional circulation (solid arrow). (B) When eddy AM flux associated with baroclinic instability (hollow arrow) is present. In this case, AM is transported by the eddy AM flux as well as the meridional circulation, so $M$ can change along the meridional circulation stream line. In this particular instance, $M$ is decreased and increased along the upper and lower branches, respectively, due to the instability, so the eddy AM transport acts as if the AM flow partly shortcuts the meridional circulation. 
A

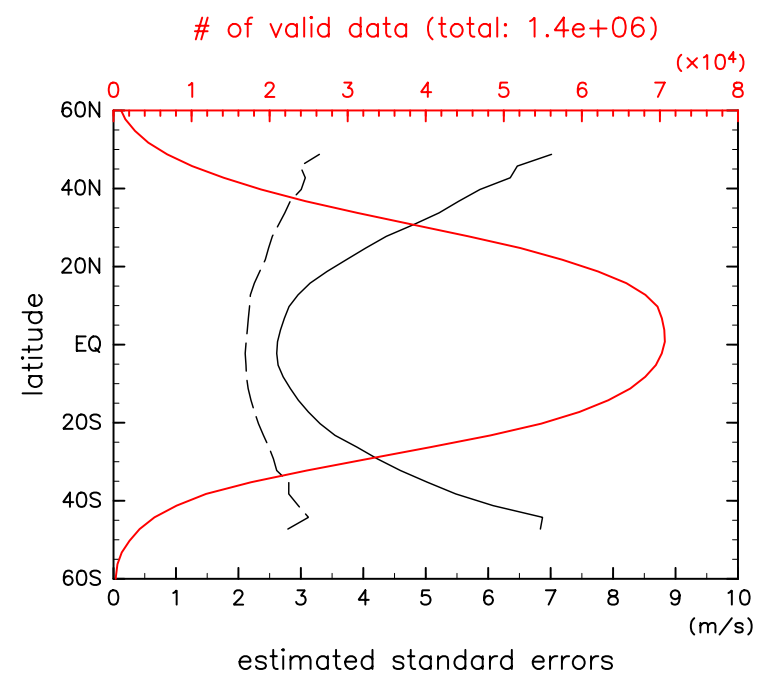

C

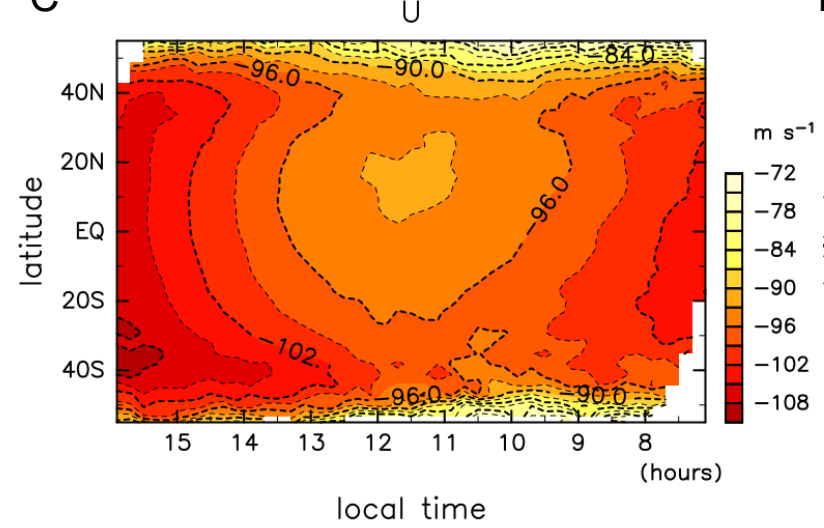

B

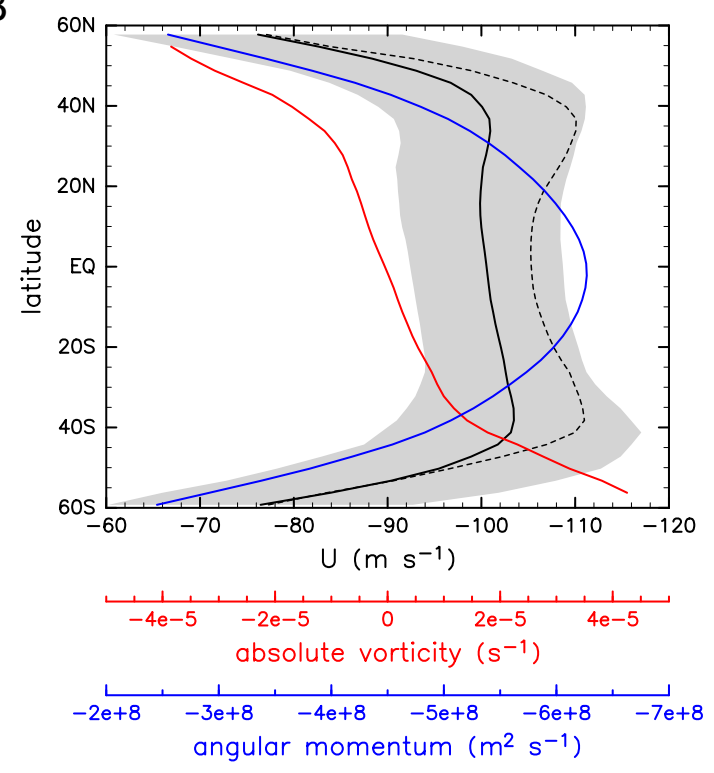

D

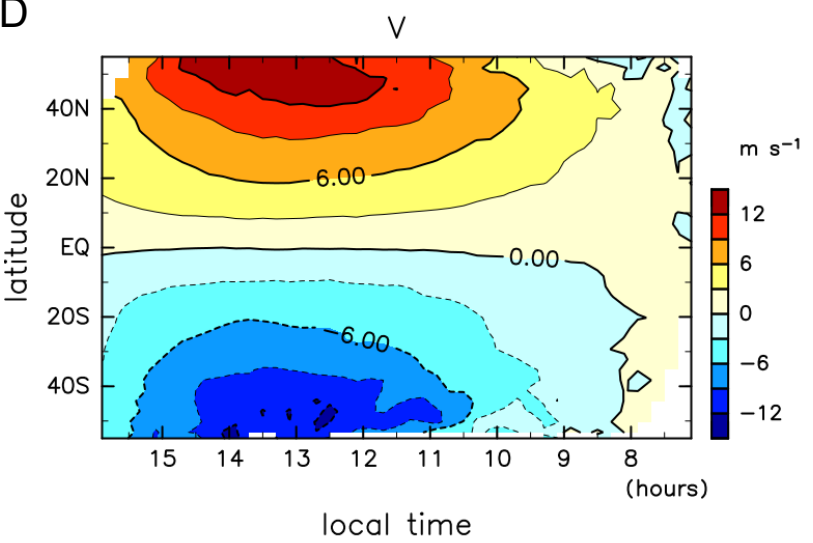

Fig. S3.

Basic statistics of the horizontal winds at the cloud top obtained from ultraviolet images taken by Akatsuki. Results from 365-nm images are shown unless otherwise stated. (A) The number of wind vectors for each latitudinal grid point with a $3^{\circ}$ interval (red curve), and the estimated standard deviations of error of the wind components after $3 \times 3$ running "tile" mean: zonal wind (black solid curve) and meridional wind (dashed curve). (B) Mean zonal wind (black solid curve) obtained by averaging over the entire period and local time over $8-9.5$ and 14 $15.5 \mathrm{~h}$. Shading indicates \pm the low-frequency variability obtained as the standard deviation of zonal wind subject to a 21 -day running-mean ( $>50 \%$ data existence required) and averaging over the designated local times. Angular momentum per unit mass (blue) and the vertical component of absolute vorticity (red) derived from the mean zonal wind. Also shown is the mean zonal wind obtained from 283-nm images (dotted). (C) Local time-latitude distribution of zonal wind averaged over the observational period $\left(\mathrm{m} \mathrm{s}^{-1}\right)$. (D) As in $\mathrm{C}$ but for meridional wind. 

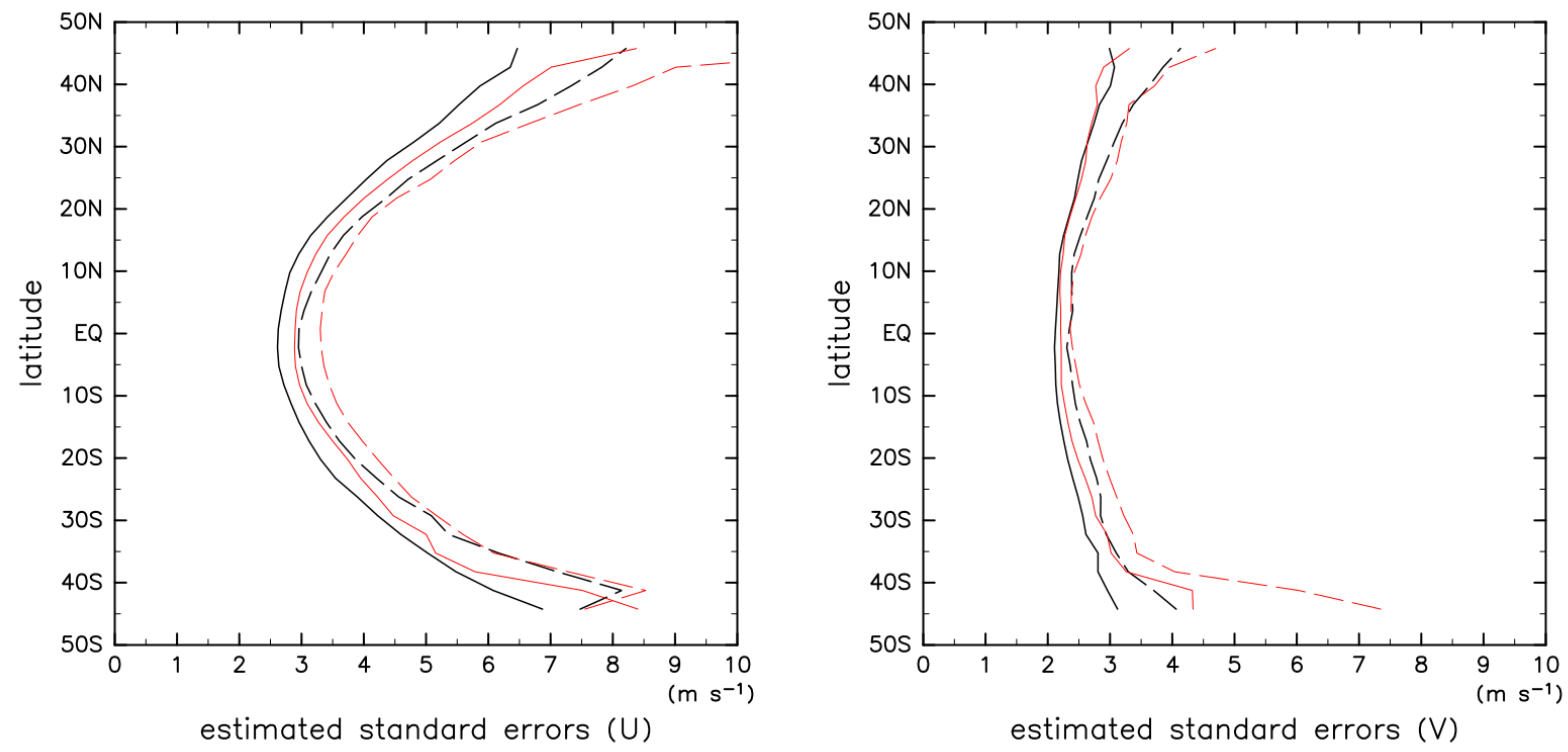

Fig. S4.

Statistics based on the Lagrangian wind differences defined in subsection S4. Left: $\sigma_{\Delta_{u}}$ at $\Delta t=4$ hours (solid) and 8 hours (dashed) for winds from the 365-nm (black) and 283-nm (red) images. Right: same but for $\sigma_{\Delta_{v}} . \sigma_{\Delta_{u}}$ and $\sigma_{\Delta_{v}}$ at $\Delta t=4$ hours are used as the estimated standard errors shown in Fig. S3A. 


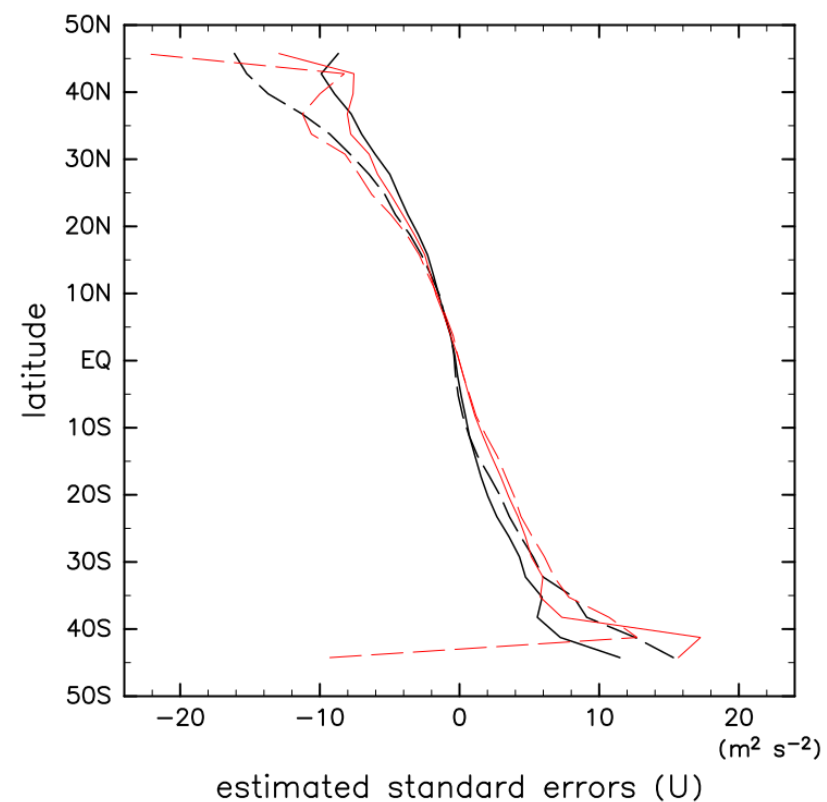

Fig. S5. Estimation of the error covariance. The halved covariance of the Lagrangian wind differences $\frac{1}{2} \mathrm{E}(\Delta u \Delta v)$ defined in subsection $\mathbf{S 6}$ when $\Delta t=4$ hours (solid) and 8 hours (dashed) for winds from the 365-nm (black) and 283-nm (red) images. The values at $\Delta t=4$ hours are used as the estimated error covariance between $u^{\prime}$ and $v^{\prime}$ to derive the AM flux shown in Fig. 2B. 

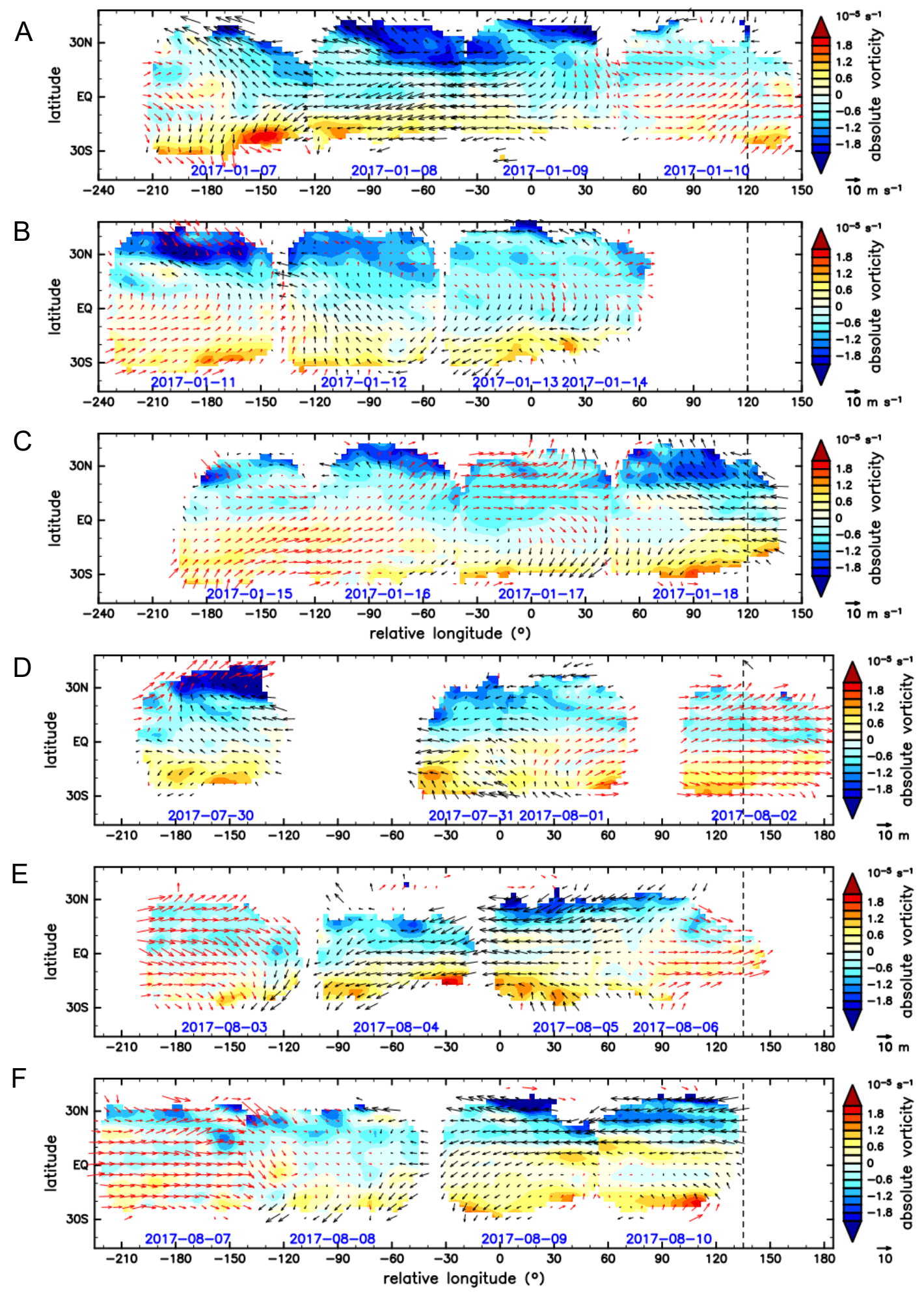

Fig. S6. Example of the spatial distribution and the temporal evolution of transient wind disturbances. The daily-mean transient winds $\left(u^{\#}, v^{\#}\right)$ over (A-C) Jan 7-18, 2017 and (D-F) July 30-Aug 10, 2017 (vectors colored in red if $u^{\prime}>0$; scale in $\mathrm{m} \mathrm{s}^{-1}$ indicated on the lowerright margins). Absolute vorticity (color shading). The longitude is based on $\tau_{L}$ and shifted by $90^{\circ}$ per day. The vertical dotted lines are shown to indicate $360^{\circ}$ from the left sides. 

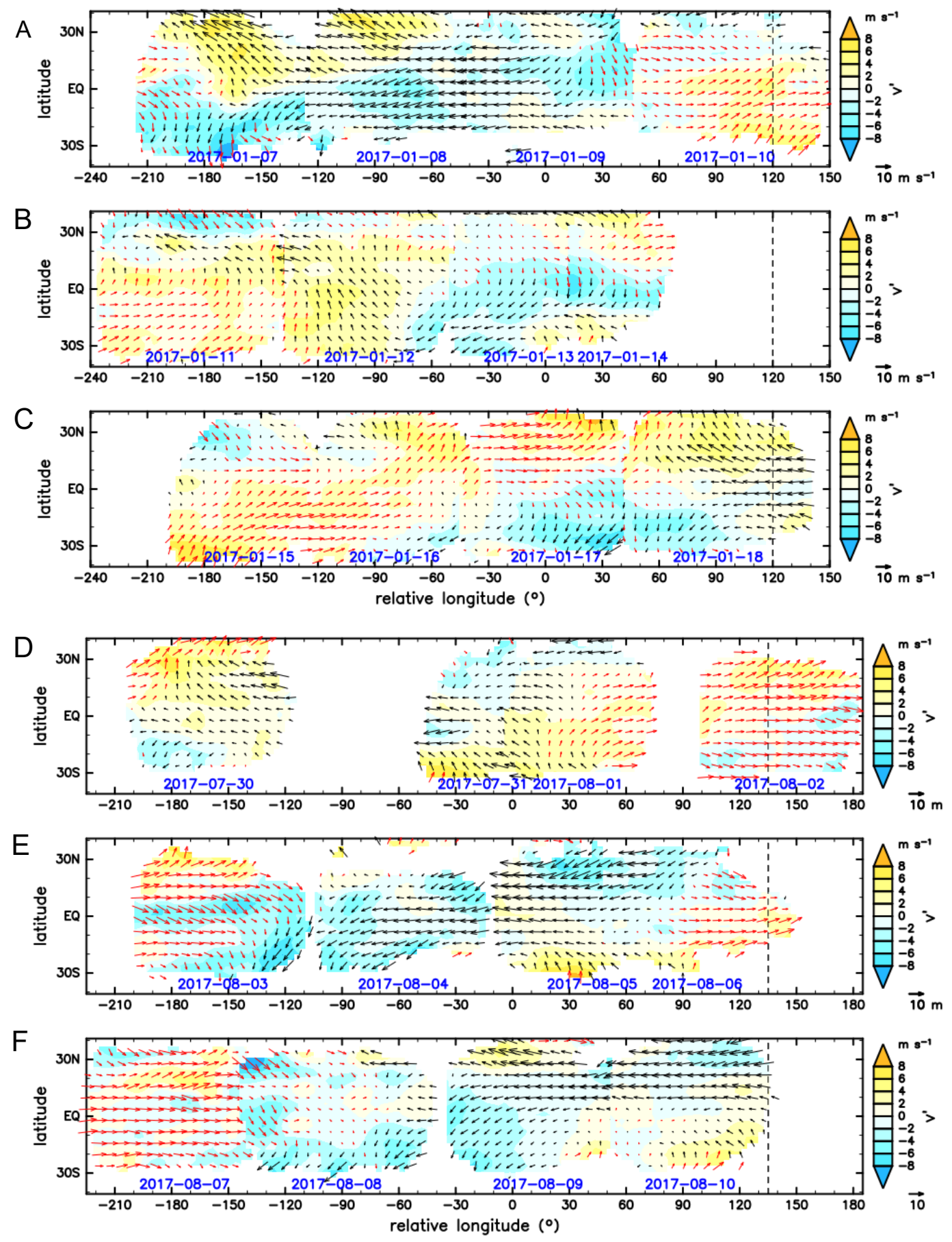

Fig. S7. As in Fig. S6 but shading is applied to $v^{\#}$. 
A

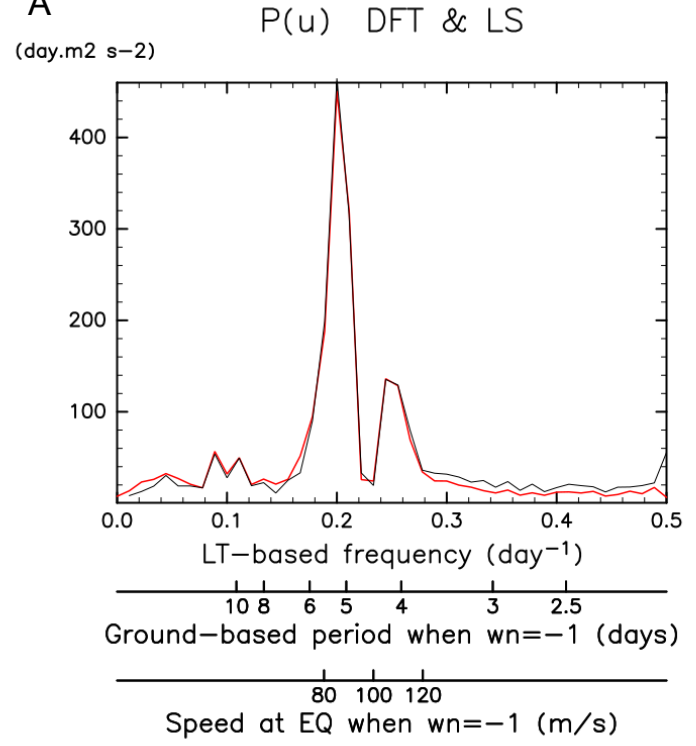

C

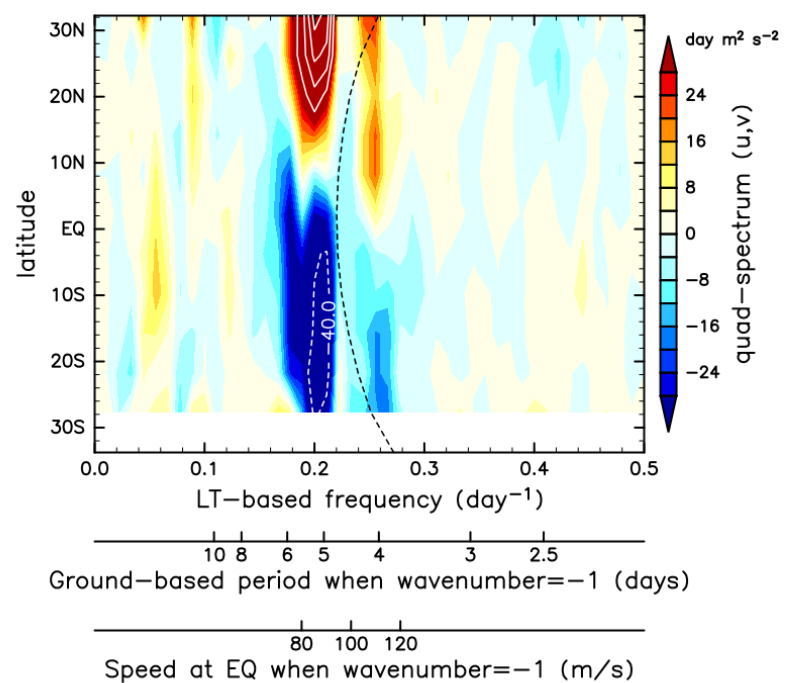

B

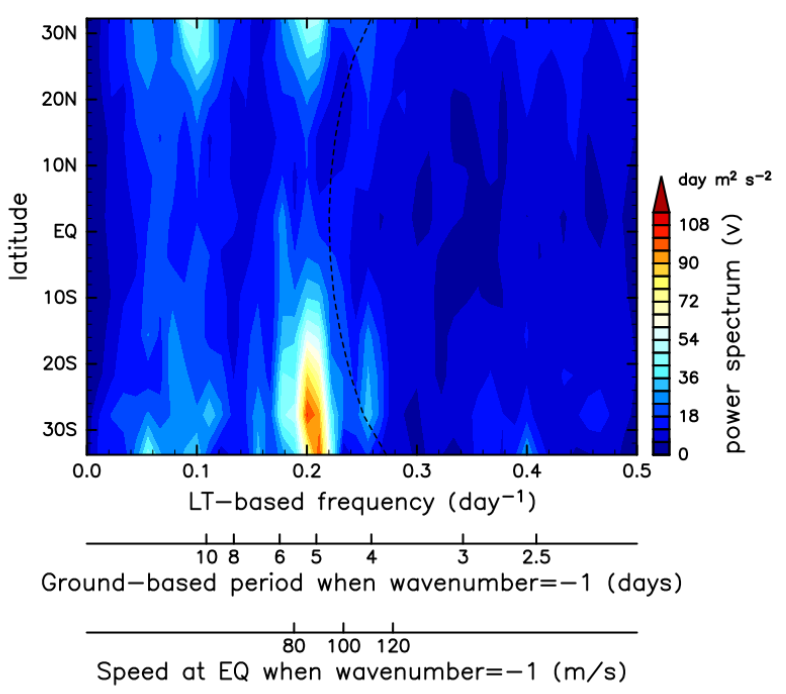

D

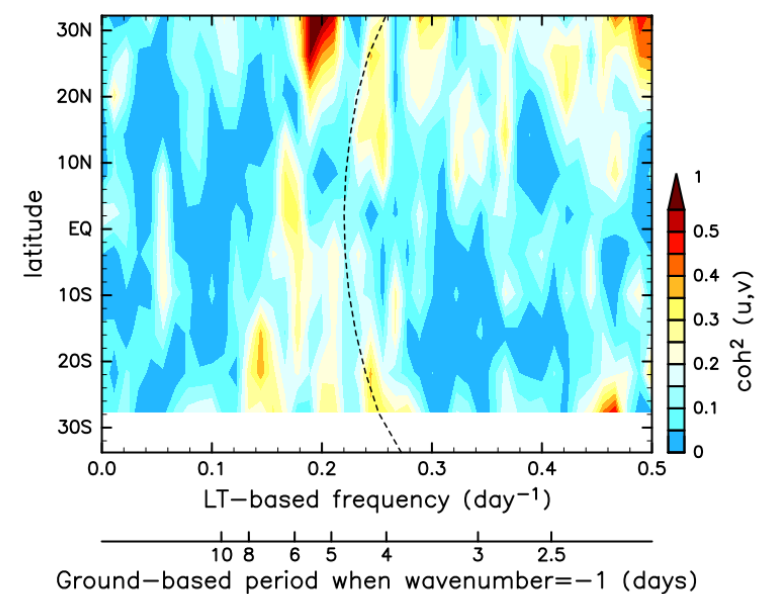

Ground-based period when wavenumber $=-1$ (days)

80100120
Speed at EQ when wavenumber $=-1(\mathrm{~m} / \mathrm{s})$

Fig. S8. Spectra and cross spectra of the daily-mean transient wind disturbances at 365-nm. (A) comparison of the Lomb-Scargle spectrum (black) and the DFT (red) spectrum of $u^{\#}$ between $5^{\circ} \mathrm{S}$ and $5^{\circ} \mathrm{N}$. (B-D) Results from DFT of daily-mean $u^{\#}$ and $v^{\#}$. (B) Power spectra of $v^{\#}\left(\mathrm{~m}^{2} \mathrm{~s}^{-2}\right.$ day). (C) As Fig. 2C but for the quadrature-spectra (the imaginary part of crossspectra). (D) Squared coherency between $u^{\#}$ and $v^{\#}$ 

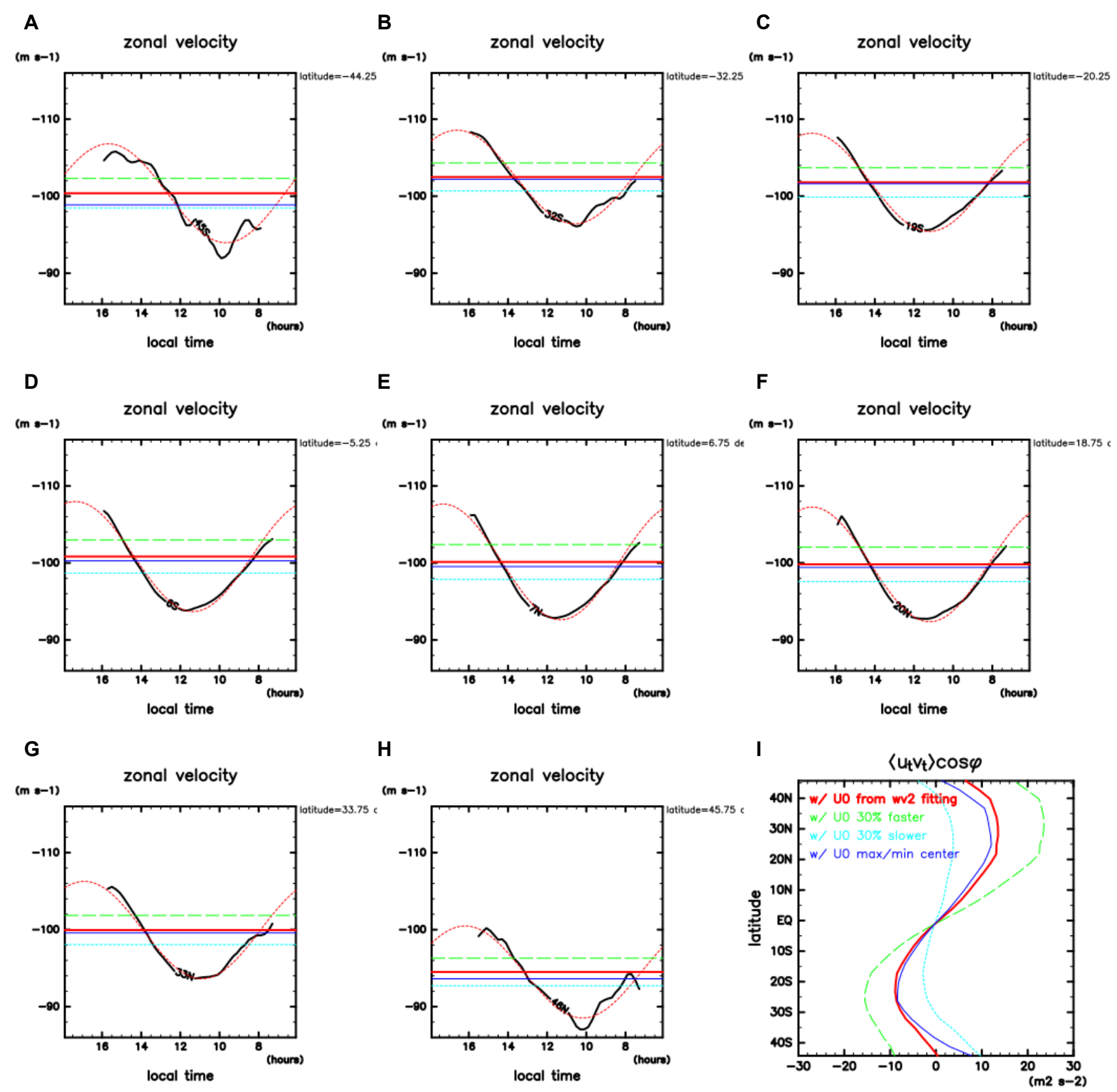

Fig. S9. Illustration of the sensitivity test to investigate the dependence of tidal angular momentum flux on the assumption of $u_{0}$. A-H: mean zonal wind $\bar{u}$ obtained from the threeyear period (black curves) at latitudes shown on their upper-right corners. The red lines indicate the values of $u_{0}$ obtained by the least-square fitting of the wavenumber- 2 sinusoidal curve. I, $\left\langle u_{t} v_{t}\right\rangle \cos \varphi$ obtained from this assumption (red curve), which is the same as the thin solid blue curve in Fig. 2A. All panels: the green and cyan lines (A-H) and curves (I) show the cases in which $u_{0}$ defined this way is shifted by \pm 0.3 times the fitting-based tidal amplitude (green: the faster; cyan: the slower). The blue lines (A-H) and curve (I) show the case in which $u_{0}$ is defined as the center in between the maximum and the minimum of $\bar{u}$. 

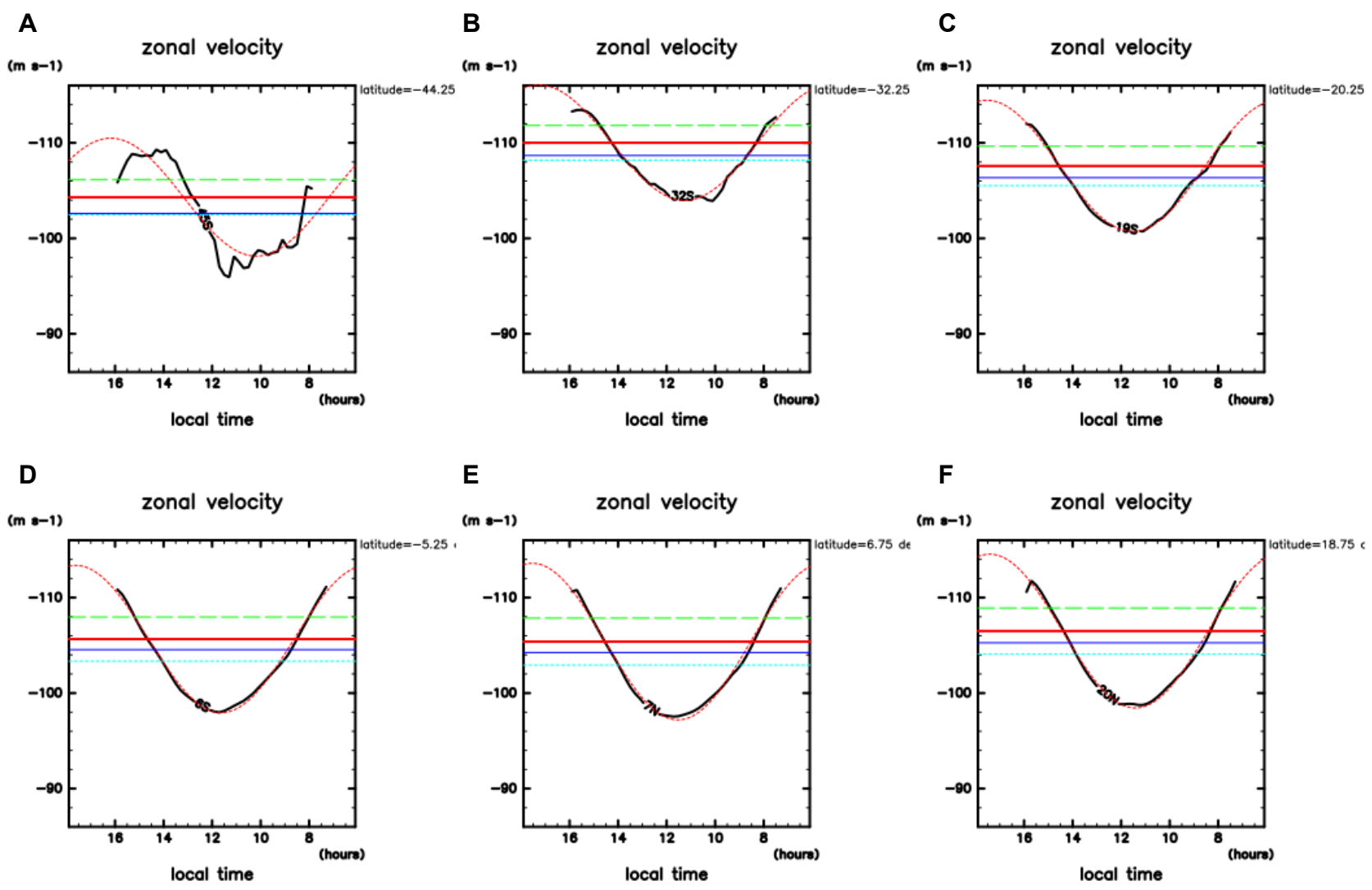

E

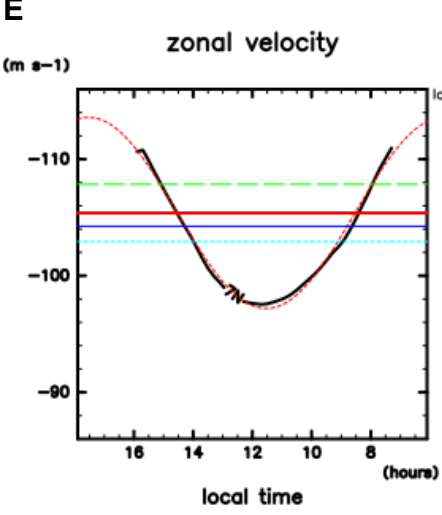

$$
\mathbf{F}
$$
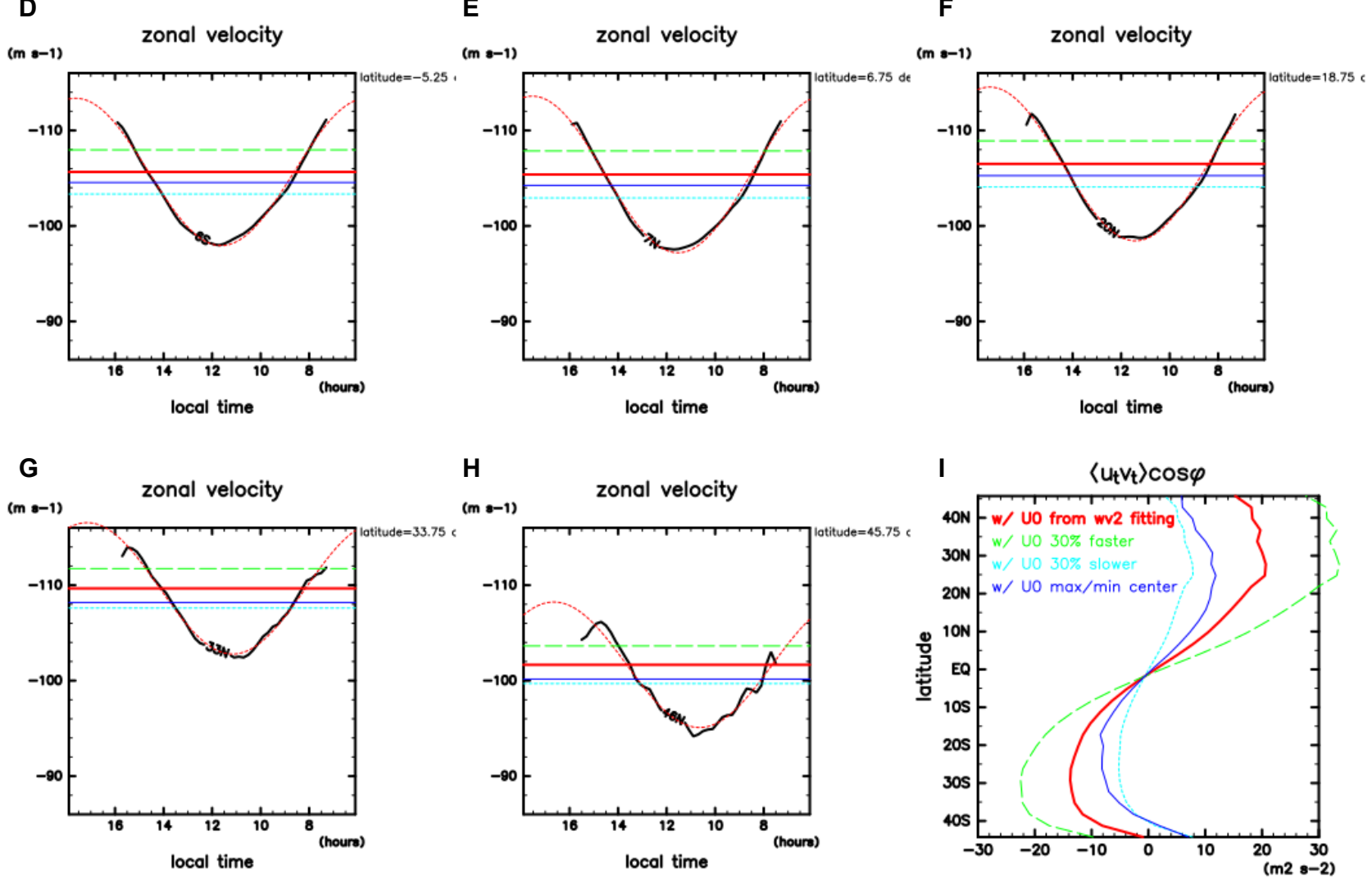

Fig. S10. As Fig. S9 but for winds obtained from 283-nm images. 

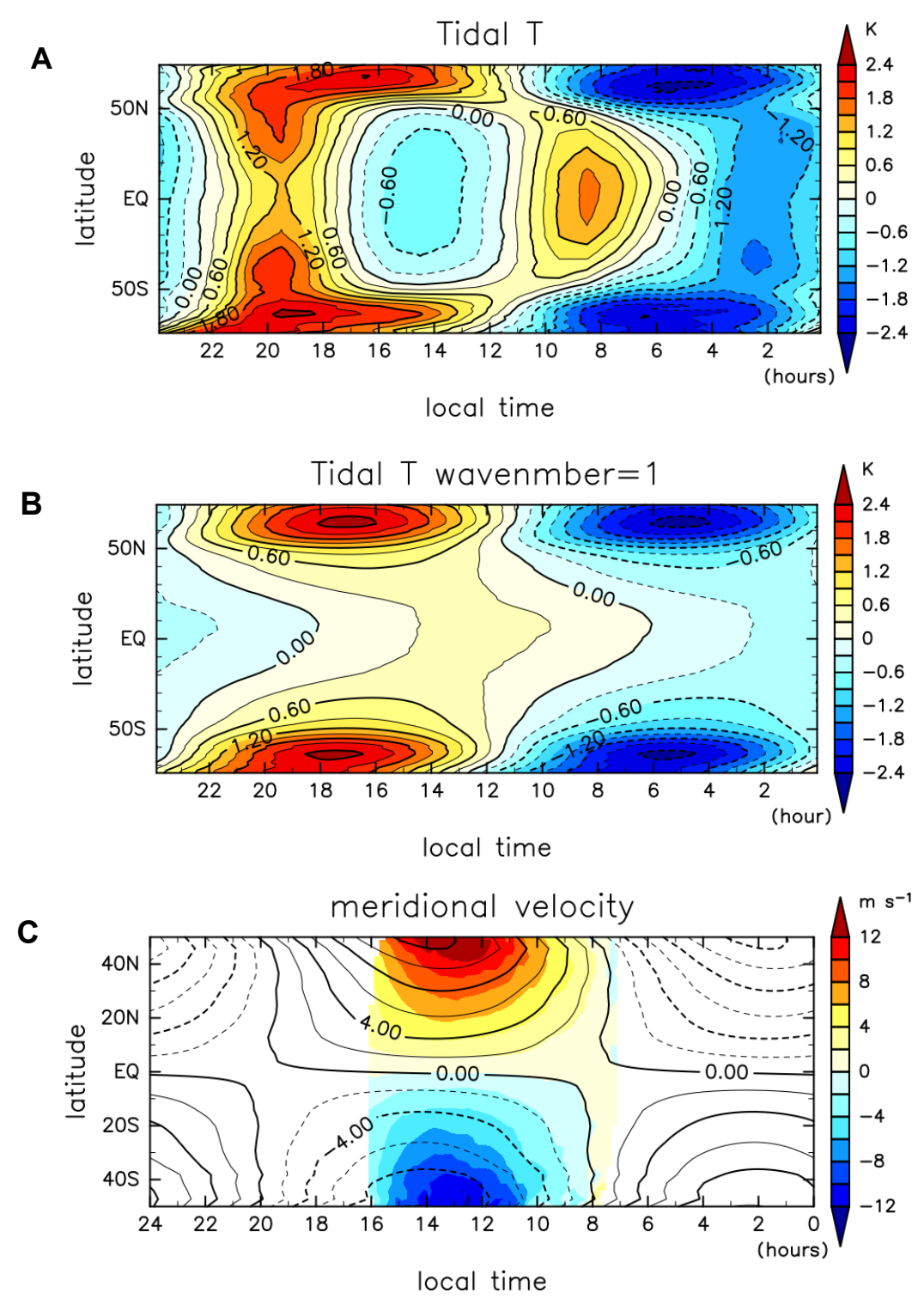

Fig. S11. Tidal temperature and meridional wind. A: the tidal component (as the deviation from zonal mean) of brightness temperature, $T_{t}(\mathrm{~K})$, derived from LIR observations when the satellite zenith angle (emission angle) is between $50^{\circ}$ and $80^{\circ}$, obtained by averaging over the three observational years. B: its zonal wavenumber-1 component. $\mathbf{C}$ : mean meridional wind $\bar{v}$ $\left(\mathrm{m} \mathrm{s}^{-1}\right)$ obtained from the UVI 365-nm images averaged over the three observational years (color-shading), and its zonal wavenumber-1 component obtained by the least-square fitting (contours) supposing $v_{0}=0$. The abscissa, LT, is shown in the decreasing order to reflect longitude (to have east to the right). See subsection $\mathbf{S 1 0}$ for more details. 


\section{Table S1.}

Downward solar flux $F(z)$ used in this study. Values between 10 and $90 \mathrm{~km}$ are taken from VIRA (39, their Figure 6-13). The value at $100 \mathrm{~km}\left(158 \mathrm{~W} \mathrm{~m}^{-2}\right)$ is assumed. The surface value $(F(0 \mathrm{~km}))$ was read as $19 \mathrm{~W} \mathrm{~m}^{-2}$ in VIRA, but it was set to 0 in our heating computation to incorporate the convective and radiative heating from the ground that absorbed solar flux.

\begin{tabular}{|l|l|l|l|l|l|l|l|l|l|l|l|l|l|}
\hline Altitude: $z(\mathrm{~km})$ & 0 & 10 & 20 & 30 & 40 & 48 & 58 & 70 & 72 & 76 & 80 & 90 & 100 \\
\hline $\begin{array}{l}\text { Downward solar } \\
\text { flux: } F\left(\mathrm{~W} \mathrm{~m}^{-2}\right)\end{array}$ & $\begin{array}{l}19 \\
(0)\end{array}$ & 20 & 24 & 30 & 40 & 41 & 57 & 136.5 & 142 & 147.5 & 150.5 & 157 & 158 \\
\hline
\end{tabular}

\section{References (34-56)}

34. M. Nakamura, et al., Overview of Venus orbiter, Akatsuki. Earth, planets and space, 63(5), 443-457 (2011).

35. M. Nakamura, et al., AKATSUKI returns to Venus. Earth Planets Space, 68:75, doi:10.1186/s40623-016-0457-6 (2016).

36. T. Horinouchi, et al., Equatorial jet in the lower to middle cloud layer of Venus revealed by Akatsuki. Nature Geoscience, 10, 646-651. doi:10.1038/ngeo3016 (2017)

37. J. Peralta, et al., Nightside Winds at the Lower Clouds of Venus with Akatsuki/IR2: Longitudinal, Local Time, and Decadal Variations from Comparison with Previous Measurements. The Astrophysical Journal Supplement Series, 239(2), 29 (2018).

38. M. G. Tomasko, L. R. Doose, P. H. Smith, A. P. Odell,. Measurements of the flux of sunlight in the atmosphere of Venus. J. Geophys. Res., 85(A13), 8167-8186 (1980).

39. V. I. Moroz, et al., Solar and thermal radiation in the Venus atmosphere. Advances in space research, 5, 197-232 (1985).

40. D. G.Andrews, J. R. Holton, C. B. Leovy, Middle Atmosphere Dynamics. (Academic press, Orland, Florida, 1987).

41. R. Hueso, J. Peralta, A. Sánchez-Lavega, Assessing the long-term variability of Venus winds at cloud level from VIRTIS-Venus Express. Icarus, 217(2), 585-598 (2012).

42. W. B. Rossow, A. D. Del Genio, S. S. Limaye, L. D. Travis, P. H. Stone, Cloud morphology and motions from Pioneer Venus images. J. Geophys. Res., 85(A13), 8107-8128 (1980).

43. F. W. Taylor, R. Beer, T. Chahine, D. J. Diner, L. S. Elson, R. D. Haskins, D. J. McCleese, J. V. Martonchik, P. E. Reichley, Structureand Meteorology of the Middle Atmosphere of Venus: Infrared Remote Sensing Fromthe Pioneer Orbiter. Journal of Geophysical Research, 85, 79638006 (1980).

44. M. Takagi, N. Sugimoto, H. Ando, Y. Matsuda, Three-dimensional structures of thermal tides simulated by a Venus GCM. J. Geophys. Res.: Planets, 123, 335-352 (2018).

45. M. Yamamoto, K. Ikeda, M. Takahashi, T. Horinouchi, Solar-locked and geographical atmospheric structures inferred from a Venus general circulation model with radiative transfer. Icarus, 321, 232-250 (2019).

46. J. D. Scargle, Studies in astronomical time series analysis. II - Statistical aspects of spectral analysis of unevenly spaced data. The Astrophysical Journal, 263, 835. https://doi.org/10.1086/160554 (1982). 
47. T. Fukuhara, et al., LIR: Longwave infrared camera onboard the Venus orbiter Akatsuki. Earth, Planets and Space, 63(9), 1009-1018 (2011).

48. N. I.Ignatiev, et al. Altimetry of the Venus cloud tops from the Venus Express observations. $J$. Geophys. Res., 114, E00B43, doi:10.1029/2008JE003320 (2008).

49. D. Crisp, Radiative forcing of the Venus mesosphere: II. thermal fluxes, cooling rates, and radiative equilibrium temperatures. Icarus, 77(2), 391-413 (1989).

50. D. Crisp, D. Titov, "The thermal balance of the Venus atmosphere." in Venus II, S. W. Bougher, D. M. Hunten, R. J. Phillips, Eds. (University of Arizona Press, Tucson, Arizona, 1997), pp 353-384.

51. Y. J.Lee, D. V. Titov, N. I. Ignatiev, S. Tellmann, M. Pätzold, G. Piccioni, The radiative forcing variability caused by the changes of the upper cloud vertical structure in the Venus mesosphere. Planetary and Space Science, 113, 298-308 (2015).

52. S.S. Limaye, Venus: Cloud level circulation during 1982 as determined from Pioneer cloud photopolarimeter images. II - Solar longitude dependent circulation. Icarus 73, 212-226 (1988).

53. A. Seiff, et al., Models of the structure of the atmosphere of Venus from the surface to 100 kilometers altitude. Advances in Space Research, 5(11), 3-58 (1985).

54. D. O. Staley, The adiabatic lapse rate in the Venus atmosphere. J. Atmos. Sci., 27(2), 219-223 (1970).

55. A. Y. Hou, R. M. Goody, Diagnostic requirements for the superrotation on Venus. J. Atmos. Sci., 42(5), 413-432 (1985).

56. M. S. Longuet-Higgins, The eigenfunctions of Laplace's tidal equation over a sphere. Philosophical Transactions of the Royal Society of London. Series A, 262(1132), 511-607 (1968). 